
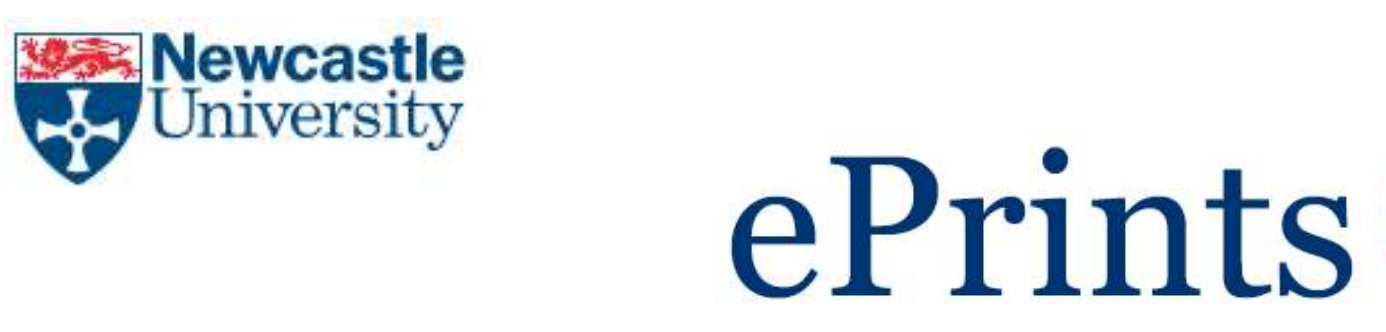

Turan O, Yigit S, Chakraborty N. Numerical investigation of mixed convection of Bingham fluids in cylindrical enclosures with heated rotating top wall. International Journal of Heat and Mass Transfer 2017, 108(Part B), 18501869.

\title{
Copyright:
}

(C) 2017. This manuscript version is made available under the CC-BY-NC-ND 4.0 license

DOI link to article:

http://dx.doi.org/10.1016/j.ijheatmasstransfer.2017.01.034

Date deposited:

$13 / 02 / 2017$

Embargo release date:

21 January 2018

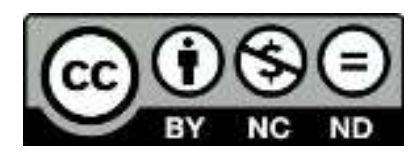

This work is licensed under a

Creative Commons Attribution-NonCommercial-NoDerivatives 4.0 International licence 


\section{Numerical investigation of mixed convection of Bingham fluids in cylindrical enclosures with heated rotating top wall}

Osman Turan $^{1,2}$, Sahin Yigit ${ }^{2}$, Nilanjan Chakraborty ${ }^{2, \bigotimes}$

${ }^{1}$ Department of Mechanical and Manufacturing Engineering, Bilecik Şeyh Edebali University, Bilecik, 11230, TURKEY

${ }^{2}$ School of Mechanical and Systems Engineering, Newcastle University, Newcastle-Upon-Tyne, NE1 7RU, UK

${ }^{\bowtie}$ corresponding author.

nilanjan.chakraborty@ncl.ac.uk 


\begin{abstract}
The steady-state laminar mixed convection of yield stress fluids obeying the Bingham model in a cylindrical enclosure with a heated rotating top cover has been numerically analysed based on axisymmetric incompressible flow simulations. Yield stress effects on heat and momentum transport have been investigated for an aspect ratio (height/radius) of unity (i.e. $A R=1$ ) for a range of different values of nominal Prandtl, Richardson and Reynolds numbers given by $10 \leq P r \leq 500,0 \leq R i \leq 1$ and $100 \leq R e \leq$ 3000. The mean Nusselt number $\overline{N u}$ has been found to decrease sharply with increasing Bingham number $B n$, but subsequently $\overline{N u}$ approaches asymptotically to a value of unity, which is indicative of conduction-driven transport. It has also been found that $\overline{N u}$ increases with increasing values of Prandtl and Reynolds numbers for both Newtonian (i.e. $B n=0$ ) and Bingham fluids. In contrast, $\overline{N u}$ decreases with increasing Ri for both Newtonian and Bingham fluids for small values of Bingham number, whereas $\overline{N u}$ remains insensitive to the variation of $R i$ for large values of Bingham number. The variation of torque coefficient $C_{T}$, which gives a quantitative measure of power consumption, has also been investigated. The torque coefficient $C_{T}$ has been found to increase with increasing $B n$ whereas it decreases with increasing $R e$. It has also been found that $C_{T}$ decreases slightly with increasing $R i$ for small values of $B n$, whereas it becomes insensitive to the variation of $R i$ for large Bingham numbers. For the fully forced convection ( $R i$ $=0$ ) case, $\operatorname{Pr}$ does not have a significant influence on $C_{T}$. However, in the case of mixed convection $C_{T}$ increases with increasing $P r$. The simulation data has been used in conjunction with a detailed scaling analysis to propose a correlation for $\overline{N u}$ for the range of $R e, R i$ and $\operatorname{Pr}$ considered here.
\end{abstract}

Keywords: Mixed convection, Bingham fluid, Rotating end wall, Reynolds number, Prandtl number, Richardson number, Bingham number 


\section{NOMENCLATURE}

\begin{tabular}{|c|c|c|}
\hline$a$ & {$[-]$} & Bridging function \\
\hline$a_{1}, a_{2}$ & {$[-]$} & Correlation parameters \\
\hline$A R$ & {$[-]$} & Aspect ratio $(A R=H / R)$ \\
\hline$b$ & {$[-]$} & Bridging function \\
\hline$b_{1}, b_{2}$ & {$[-]$} & Correlation parameters \\
\hline$B n$ & {$[-]$} & Bingham number \\
\hline$c_{p}$ & {$[\mathrm{~J} / \mathrm{kgK}]$} & Specific heat at constant pressure \\
\hline$C_{T}$ & {$[-]$} & Torque coefficient \\
\hline$e_{a}$ & {$[-]$} & Relative error \\
\hline$f_{1}, f_{2}, f_{3}$ & {$[-]$} & Functions \\
\hline$g$ & {$\left[\mathrm{~m} / \mathrm{s}^{2}\right]$} & Gravitational acceleration \\
\hline$G r$ & {$[-]$} & Grashof number \\
\hline$h$ & {$\left[\mathrm{~W} / \mathrm{m}^{2} \mathrm{~K}\right]$} & Heat transfer coefficient \\
\hline$H$ & {$[\mathrm{~m}]$} & Height of cylindrical enclosure \\
\hline$k$ & {$[\mathrm{~W} / \mathrm{mK}]$} & Thermal conductivity \\
\hline$k_{0}, k_{1}$ & {$[-]$} & Correlation parameters \\
\hline$m_{0}, m_{1}$ & {$[-]$} & Correlation parameters \\
\hline $\mathrm{Nu}$ & {$[-]$} & Nusselt number \\
\hline$\overline{N u}$ & {$[-]$} & Mean Nusselt number \\
\hline$p$ & {$[-]$} & Theoretical order of accuracy \\
\hline $\operatorname{Pr}$ & {$[-]$} & Prandtl number \\
\hline$q$ & {$\left[\mathrm{~W} / \mathrm{m}^{2}\right]$} & Heat flux \\
\hline$R$ & {$[\mathrm{~m}]$} & Radius of cylindrical enclosure \\
\hline$R a$ & {$[-]$} & Rayleigh number \\
\hline $\operatorname{Re}$ & {$[-]$} & Reynolds number \\
\hline$R i$ & {$[-]$} & Richardson number \\
\hline$T$ & {$[\mathrm{~K}]$} & Temperature \\
\hline$U$ & $(\mathrm{~m} / \mathrm{s})$ & Characteristic velocity scales in radial direction \\
\hline$V$ & $(\mathrm{~m} / \mathrm{s})$ & Characteristic velocity scales in tangential direction \\
\hline$V_{\theta}$ & {$[-]$} & Non-dimensional swirl velocity $\left(V_{\theta}=v H / \alpha\right)$ \\
\hline$\alpha$ & {$\left[\mathrm{m}^{2} / \mathrm{s}\right]$} & Thermal diffusivity \\
\hline$\beta$ & {$[1 / \mathrm{K}]$} & Coefficient of thermal expansion \\
\hline$\dot{\gamma}$ & {$[1 / \mathrm{s}]$} & Shear rate \\
\hline$\delta, \delta_{t h}$ & {$[\mathrm{~m}]$} & Hydrodynamic and thermal boundary layer thickness \\
\hline$\theta$ & {$[-]$} & Non- dimensional temperature $\left(\theta=\left(T-T_{\mathrm{C}}\right) /\left(T_{\mathrm{H}}-T_{\mathrm{C}}\right)\right)$ \\
\hline$\mu$ & {$\left[\mathrm{Ns} / \mathrm{m}^{2}\right]$} & Plastic viscosity \\
\hline
\end{tabular}




$\begin{array}{lll}\mu_{\text {yield }} & {\left[\mathrm{Ns} / \mathrm{m}^{2}\right]} & \text { Yield viscosity } \\ v & {\left[\mathrm{~m}^{2} / \mathrm{s}\right]} & \text { Kinematic viscosity } \\ \rho & {\left[\mathrm{kg} / \mathrm{m}^{3}\right]} & \text { Density } \\ \tau & {\left[\mathrm{N} / \mathrm{m}^{2}\right]} & \text { Shear stress } \\ \tau_{y} & {\left[\mathrm{~N} / \mathrm{m}^{2}\right]} & \text { Yield stress } \\ \phi & {[-]} & \text { General primitive variable } \\ \Omega & {[1 / \mathrm{s})} & \text { Angular velocity } \\ \psi & {\left[\mathrm{m}^{2} / \mathrm{s}\right]} & \text { Stream function } \\ \Psi & {[-]} & \text { Non-dimensional stream function }(\Psi=\psi / \alpha)\end{array}$

\section{Subscripts}

$B n=0$

$C$

eff

$H$

$\max$

$r$

ref

$w f$

$z$

$\theta$

Special characters

$\Delta T \quad[\mathrm{~K}]$

$\Delta_{\text {min,cell }} \quad[\mathrm{m}]$

$r$

$[-]$
Newtonian fluid case

Cold wall

Effective value

Hot wall

Maximum value

Radial direction

Reference value

Condition of the fluid in contact with the wall

Axial direction

Tangential direction

Difference between hot and cold wall temperature $\left(=\left(T_{\mathrm{H}}-T_{\mathrm{C}}\right)\right)$

Minimum cell distance

Grid expansion ratio 


\section{INTRODUCTION}

Mixing and heat transfer play pivotal roles in several engineering applications relevant to chemical processing, bio-chemical synthesis, polymer processing, food preparation, modern agriculture and many other related industries. Augmentation of mixing and heat transfer in laminar flow conditions can be challenging but it is possible to enhance the rates of heat transfer and mixing by rotating one of the covers of a cylindrical container to produce a swirling flow. Although superficially it seems to be a simple process, the heat transfer rate in this configuration depends on many parameters, such as the container geometry and rotational speed of cover. Flow in cylindrical enclosures with a rotating cover has been extensively analysed owing to its wide-ranging roles in the aforementioned engineering applications. However, the majority of these analyses have been conducted only for Newtonian Fluids (where the viscous stress is directly proportional to strain rate). Vogel [1,2], Ronnenberg [3], and Bertela and Gori [4] pioneered the analysis of this configuration for Newtonian fluids. The findings of these studies [1-4] were confirmed and extended by Escudier [5] based on an experimental analysis where the stability criterion for vortex breakdown was identified in terms of aspect ratio $(\mathrm{AR}=$ height/radius $=H / R)$ and Reynolds number $\left(\Omega R^{2} / v\right)$. In addition to these experimental studies, several numerical investigations [6-10] involving Newtonian fluids have been carried out. Lee and Hyun [8] analysed the effects of Prandtl number on heat transfer rate in this configuration and revealed that Prandtl number has an important influence on the heat transfer characteristics and advective transport has been found to strengthen with increasing Prandtl number. Iwatsu [9] investigated the effects of Reynolds and Richardson numbers in the range of $100 \leq R e \leq 3000$ and $0 \leq R i \leq 1$ for $P r=1$ on the flow pattern and heat transfer rate for swirling flows in cylindrical enclosures with an aspect ratio of unity (i.e. $A R=H / R=1)$ and a heated rotating top wall. The analysis by Iwatsu [9] revealed that advective (diffusive) transport strengthens (weakens) and, accordingly, the mean Nusselt number decreases with increasing Richardson number. Compared to Newtonian fluids, relatively limited efforts have been directed to the analysis of mixed convection of nonNewtonian fluids (where the viscous stress is not proportional to the strain rate). Although both water and air, being the most abundant fluids on the planet, are Newtonian fluids, and it is logical that most previous 
work concentrated on such Newtonian fluids, virtually all synthetic, food-based and biological fluids (e.g. detergents, toothpaste, cosmetics, ketchup, custard, blood) are non-Newtonian in character. Some electrorheological/magneto-rheological fluids exhibit yield stress and behave only as fluids once a threshold stress is surpassed. It is possible to modulate the yield stress by using an electrical/magnetic field in electro-rheological/magneto-rheological fluids. Thus, from an engineering perspective, the knowledge of mixed convection of more rheologically complex fluids than water or air is essential.

One of the first investigations related to swirling flows for non-Newtonian fluids was performed for flows over a rotating disk, which is commonly referred to as the von-Karman flow [11-13]. Most analyses on the non-Newtonian fluid flow in cylindrical enclosures with a rotating cover were carried out for viscoelastic fluids $[14,15]$. Escudier and Cullen [14] experimentally analysed cylindrical enclosures with a rotating top cover for shear-thinning viscoelastic fluids, and reported that the vortex structure is different from the Newtonian fluid case and an intense toroidal vortex that drives a secondary low intensity vortex was produced in the vicinity of the rotating cover. Stokes and Boger [15] proposed a regime diagram for flow stability based on Reynolds and Elasticity numbers for viscoelastic fluids in cylindrical enclosures with a rotating cover. A recent experimental analysis of heat transfer under elastic turbulence of viscoelastic fluids within cylindrical enclosures with a rotating top cover was reported by Traore et al. [16]. It was found that the heat transfer rate under elastic turbulence might locally increase by up to four times as compared to the purely conduction regime. It was indicated by Traore et al. [16] that the elastic turbulence can augment the heat transport rate in situations where the inertial turbulence is difficult to obtain, such as in the case of microscopic flows.

Analysis of power-law fluids in cylindrical enclosures with a rotating cover was carried out by Böhme et al. [17]. The influence of shear-thinning on vortex breakdown (observed before by Vogel [1,2] and Escudier [5] for Newtonian fluids) in cylindrical enclosures with a rotating cover was experimentally and numerically analysed by Böhme et al. [17]. They constructed an aspect ratio - Reynolds number $(A R-R e)$ 
diagram, representing the domain of vortex breakdown for shear-thinning fluids. A few recent analyses [18-20] have concentrated on rotating disk configuration for yield stress fluids. The mass transfer of yield stress fluids in such applications has recently been investigated by Rashaida et al. [18] and a semianalytical approach for analysing swirling flow of Bingham fluids over a rotating disk has been reported by Ahmadpour and Sadegyhy [19] and Guha and Sengupta [20]. However, to date, there is no analysis in the existing literature that deals with flow structure and heat transfer characteristics of yield stress fluids in a cylindrical enclosure with a rotating end wall, in spite of its application in many chemical and food industries. For this purpose, the present study focuses on a detailed analysis of the heat transfer characteristics of yield stress fluids obeying the Bingham model in cylindrical enclosures with a rotating heated top cover, which is schematically shown in Fig. 1. An extensive parametric analysis has been conducted to analyse the effects of yield stress on heat and momentum transport for a range of nominal values of Prandtl, Richardson and Reynolds numbers (definitions are provided in Section 2) given by $10 \leq$ $\operatorname{Pr} \leq 500,0 \leq R i \leq 1$ and $100 \leq R e \leq 3000$, respectively. The simulation data in turn has been utilised to develop a correlation for the mean Nusselt number, $\overline{N u}$, based on a detailed scaling analysis for the range of $P r, R i, R e$ and $B n$ considered in this analysis. In this respect, the main objective of the present paper is to demonstrate the influences of Bingham, Reynolds, Richardson and Prandtl numbers on the heat and momentum transport in the case of mixed convection of Bingham fluids in a cylindrical enclosure with a heated rotating top wall for an aspect ratio of unity (i.e. $A R=H / R=1$ ).

\section{MATHEMATICAL BACKGROUND}

\subsection{Constitutive equations}

A number of empirical models have been proposed for describing the interrelation between shear stress and strain rate in yield stress fluids. In this study, the Bingham model [21] is used, which is the simplest model to describe the relationship between shear stress and strain rate in yield stress fluids. The mathematical expression of the Bingham model can be expressed as: 


$$
\begin{array}{ll}
\underline{\underline{\gamma}}=0 & \text { for } \tau \leq \tau_{y} \\
\underline{\underline{\tau}}=\left(\mu+\frac{\tau_{y}}{\dot{\gamma}}\right) \underline{\dot{\gamma}} & \text { for } \tau>\tau_{y}
\end{array}
$$

where $\underline{\underline{\gamma}}$ and $\underline{\underline{\tau}}$ are the tensor of the strain rate and shear stress, respectively, $\tau_{y}$ is the yield stress and $\mu$ is the so-called plastic viscosity of the yielded fluid. The quantities $\tau$ and $\dot{\gamma}$ are defined based on the second invariants of the stress and the rate of strain tensors, respectively:

$$
\begin{aligned}
& \tau=\left[\frac{1}{2} \underline{\underline{\tau}}: \underline{\tau}\right]^{1 / 2} \\
& \dot{\gamma}=\left[\frac{1}{2} \underline{\underline{\gamma}}: \dot{\underline{\gamma}}\right]^{1 / 2}
\end{aligned}
$$

O'Donovan and Tanner [22] used the bi-viscosity model to mimic the stress-shear rate characteristics for a Bingham fluid in the following manner:

$$
\begin{array}{ll}
\underline{\underline{\tau}}=\mu_{\text {yield }} \underline{\underline{\underline{\gamma}}} & \text { for } \dot{\gamma} \leq \frac{\tau_{y}}{\mu_{\text {yield }}} \\
\underline{\underline{\tau}}=\tau_{y}+\mu\left[\underline{\underline{\gamma}}-\frac{\tau_{y}}{\mu_{\text {yield }}}\right] & \text { for } \dot{\gamma}>\frac{\tau_{y}}{\mu_{\text {yield }}}
\end{array}
$$

where $\mu_{\text {yield }}$ is the yield viscosity.

\subsection{Governing equations and boundary conditions}

For the present analysis the flow is considered to be laminar, incompressible, steady and axisymmetric (i.e. two -dimensional). For steady-state incompressible axisymmetric swirling flows the conservation equations in the cylindrical coordinate system take the following form:

\section{Mass conservation equation}

$\frac{\partial u}{\partial r}+\frac{u}{r}+\frac{\partial w}{\partial z}=0$

\section{Momentum conservation equations}

$r: \quad \rho\left(u \frac{\partial u}{\partial r}-\frac{v^{2}}{r}+w \frac{\partial u}{\partial z}\right)=-\frac{\partial p}{\partial r}+\frac{\partial \tau_{z r}}{\partial z}+\frac{\partial \tau_{r r}}{\partial r}+\frac{\tau_{r r}-\tau_{\theta \theta}}{r}$ 


$$
\begin{array}{ll}
\theta: & \rho\left(u \frac{\partial v}{\partial r}+\frac{u v}{r}+w \frac{\partial v}{\partial z}\right)=\frac{\partial \tau_{r \theta}}{\partial r}+\frac{\partial \tau_{z \theta}}{\partial z}+\frac{2 \tau_{r \theta}}{r} \\
z: & \rho\left(u \frac{\partial w}{\partial r}+w \frac{\partial w}{\partial z}\right)=-\frac{\partial p}{\partial z}+\rho g \beta\left(T-T_{r e f}\right)+\frac{1}{r} \frac{\partial}{\partial r}\left(r \tau_{r z}\right)+\frac{\partial \tau_{z z}}{\partial z}
\end{array}
$$

Energy conservation equation

$\rho c_{p}\left(u \frac{\partial T}{\partial r}+w \frac{\partial T}{\partial z}\right)=k\left(\frac{1}{r} \frac{\partial T}{\partial r}+\frac{\partial^{2} T}{\partial r^{2}}+\frac{\partial^{2} T}{\partial z^{2}}\right)$

where $T_{r e f}$ is the reference temperature for evaluating the buoyancy term $\rho g \beta\left(T-T_{r e f}\right)$ in the momentum conservation equation in the vertical direction, and here $T_{r e f}$ is taken to be the cold (bottom) cover temperature $T_{c}$. In addition, thermo-physical properties (thermal conductivity, specific heat, plastic viscosity, yield stress, etc.) are assumed to be constant and independent of temperature in this analysis for the sake of simplicity.

The numerical investigation is carried out in an axisymmetric cylindrical container with a rotating top cover, which is schematically shown in Fig. 1. The aspect ratio $(A R=H / R)$ of the cylindrical container is considered to be unity (i.e. $A R=H / R=1$ ). The bottom and top covers of the cylindrical enclosure are kept at different temperatures $\left(T_{C}<T_{H}\right)$, while the cylindrical surface is considered to be adiabatic in nature. The temperature difference between the top and bottom covers is kept small enough to ensure that Boussinesq approximation remains valid. The velocity components are identically zero owing to the noslip conditions and the impenetrability of the surface of the container.

\subsection{Non-dimensional numbers}

In the present study, the flow and heat transfer characteristics of Bingham fluids in cylindrical containers with a rotating heated top cover are compared to the corresponding Newtonian fluid cases with the same nominal values of Reynolds, Richardson and Prandtl numbers. 
In the case of mixed convection, the ratio of inertial to viscous forces needs to be considered for the forced convection component and this ratio can be characterised in terms of a nominal Reynolds number $R e$, which can be defined as:

$$
R e=\frac{\rho \Omega R^{2}}{\mu}
$$

Buoyancy force also plays an important role in the overall heat transfer in mixed convection and it is characterised by the Grashof number, which represents the ratio of buoyancy to viscous forces, in the following manner:

$$
G r=\frac{\rho^{2} g \beta \Delta T H^{3}}{\mu^{2}}
$$

Another important non-dimensional number is the Rayleigh number, $R a$, which demonstrates that the ratio of the strengths of thermal transports owing to buoyancy to thermal diffusion, which is defined here in the following manner:

$$
R a=\frac{\rho^{2} c_{p} g \beta \Delta T H^{3}}{\mu k}=G r P r
$$

where $P r$ is the Prandtl number, which is defined as:

$$
\operatorname{Pr}=\frac{\mu c_{p}}{k}
$$

The Prandtl number depicts the ratio of momentum diffusion to thermal diffusion. The Prandtl number can also be taken to represent the ratio of the hydrodynamic boundary layer thickness to the thermal boundary layer thickness.

The dimensionless parameter used to evaluate the relative importance of the natural to forced convection is the Richardson number, which is defined as:

$$
R i=\frac{G r}{R e^{2}}=\frac{g \beta \Delta T H^{3}}{\Omega^{2} R^{4}}
$$

The ratio of yield stress to plastic viscous stress, which is characterised by the Bingham number, $B n$, is defined in the following manner: 


$$
B n=\frac{\tau_{y}}{\mu \Omega}
$$

From a practical point of view, the power consumption is one of the most important parameters in the design of a mixing system. Power consumption magnitude is closely associated with the torque applied to rotate the cover and it is usually quantified in terms of a dimensionless number called the torque coefficient, $C_{T}$. The torque coefficient, $C_{T}$, is defined in the present study in the following manner:

$$
C_{T}=\frac{\int_{0}^{R}\left(\left.\tau_{z \theta}\right|_{z=H} 2 \pi r\right) r d r}{\rho \Omega^{2} R^{5}}
$$

The rate of convective heat transfer is often characterised by the heat transfer coefficient $h$, which is expressed in non-dimensional form in terms of the Nusselt number, $N u$, as:

$$
N u=\frac{h R}{k}
$$

and the heat transfer coefficient $h$ is defined as:

$$
h=\left|-k \frac{\partial T}{\partial z}\right|_{w f} \times \frac{1}{T_{\text {wall }}-T_{\text {reff }}} \mid
$$

where subscript 'wf' refers to the condition of the fluid in contact with the wall, $T_{\text {wall }}$ is the wall temperature and $T_{r e f}$ is the appropriate reference temperature, which can be taken to be $T_{C}\left(T_{H}\right)$ for the hot (cold) wall.

It is worth noting that viscosity varies throughout the domain in Bingham fluid flows and thus an effective viscosity can be expressed as $\mu_{e f f}=\tau_{y} / \dot{\gamma}+\mu$, which might be more representative of the viscous action within the flow than the constant plastic viscosity $\mu$. Therefore, the non-dimensional numbers could have been defined more appropriately if $\mu_{\text {eff }}$ was used instead of $\mu$. However, $\dot{\gamma}$ is expected to show local variations in the flow domain so using a single characteristic value in the definitions of the nondimensional numbers may not yield any additional benefit in comparison to the definitions given by Eqs. (10)-(15). 


\subsection{Numerical implementation, grid-independency and benchmarking}

In this study, a commercial package ANSYS-FLUENT, which has previously been used successfully for simulations of both non-Newtonian [23] and Newtonian fluids [10], was used in order to solve the conservation equations of mass, momentum and energy using a finite-volume method. In this framework, a second-order up-wind scheme is used for the convective terms. Coupling of pressure and velocity is achieved using the well-known SIMPLE (Semi-Implicit Method for Pressure-Linked Equations) algorithm [24]. The convergence criteria in FLUENT were set to $10^{-9}$ for all the relative (scaled) residuals.

Three different non-uniform meshes M1 $(50 \times 50)$, M2 $(100 \times 100)$ and M3 $(200 \times 200)$ have been investigated, and the details of these meshes have been provided in Table 1 where the normalised minimum grid spacing $\Delta_{m i n, c e l l} / R$ and grid expansion ratio $r_{e}$ are provided. The numerical uncertainty for the mean Nusselt number $\overline{N u}=\int_{0}^{H} N u d z / H$ and maximum non-dimensional stream function $\Psi_{\max }(=$ $\left.\psi_{\max } / \alpha\right)$ in case of the Newtonian and Bingham fluids for $R e=1000$ and $R i=0.1$ at $\operatorname{Pr}=500$ are shown in Table 2. Table 2 highlights that all the numerical uncertainty levels between M1 $(200 \times 200)$ and M2 $(100 \times 100)$ are under $1 \%$ for both Bingham and Newtonian fluids. Based on this analysis, the simulations were conducted using mesh M2 $(100 \times 100)$, which was found to be sufficient for providing high accuracy and computational efficiency.

In addition to the aforementioned grid-independency study, the simulation results for Newtonian fluids have also been compared with respect to the data reported by Iwatsu [9] for different Richardson and Reynolds numbers values at $\operatorname{Pr}=1$. As shown in Fig. 2, the present simulation results remain in excellent agreement with the corresponding benchmark values reported by Iwatsu [9]. The numerical scheme used here was previously validated earlier for laminar natural convection of Bingham fluids in square enclosures, and interested readers are referred to Ref. [25] for further information in this regard. 


\subsection{The effects of yield viscosity and initial condition}

The choice of the ratio of the yield viscosity to plastic viscosity, $\mu_{y i e l d} / \mu$, plays a crucial role in the implementation of bi-viscosity regularisation for Bingham fluid flows. O’Donovan and Tanner [22] reported that the yield viscosity should be $10^{3}$ times the plastic viscosity to perform effectively in a squeeze film problem. On the other hand, this ratio was taken to be $10^{4}$ by Turan et al. [25] for studying natural convection in a square enclosure. In a recent paper, where von-Karman's swirling flow on a rotating disk was studied, Guha and Sengupta [20] took the value of $\mu_{y i e l d} / \mu$ to be $5 \times 10^{4}$. These indicate that the optimal ratios of the yield viscosity to plastic viscosity may change depending on the problem. Therefore, in order to assess the sensitivity of $\mu_{\text {yield }}$, a number of simulations were carried out for different values of $\mu_{y i e l d} / \mu$ and it was observed that the variation of $\overline{N u}$ with $B n$ shows some unphysical non-monotonic trend for $\mu_{y i e l d} / \mu<10^{7}$. However, there is a good quantitative agreement between the results of $\mu_{\text {yield }} / \mu=10^{7}$ and $\mu_{\text {yield }} / \mu=10^{8}$. Given this agreement, $\mu_{\text {yield }} / \mu=10^{8}$ is considered for this analysis to ensure a high level of accuracy.

In addition to the sensitivity analysis of yield viscosity, the effect of initial conditions on the numerical simulations was also checked. For this purpose, some simulations were carried out for two types of initial conditions. In one set of simulations, the quiescent flow condition is used for the initial condition, whereas the steady-state simulation results obtained for a smaller value of $B n$ is used as the initial condition for the cases in the second methodology [26]. These two methodologies have recently been referred to as the "quiescent flow" and "established flow" initial conditions, respectively, by Yigit et al. [26]. An unphysical variation of $\overline{N u}$ with $B n$ (where the $\overline{N u}$ increases with increasing $B n$ without any change in flow pattern whereas a scaling analysis reveals that $\overline{N u}$ is expected to decrease with an increase in $B n$ ) is observed in the case of quiescent flow initial conditions, while the expected trend (where the $\overline{N u}$ decreases with increasing $B n$ for a given flow pattern) is obtained for established flow initial conditions. However, this unexpected trend for quiescent flow initial conditions disappears when the more refined mesh is used 
close to the wall. An inadequate resolution of boundary layer yields an inaccurate strain rate field, which in turn affects the viscosity distribution within the domain according to the biviscosity regularisation. The above behaviour was not observed for the established flow boundary conditions, which suggests that the simulation results are more sensitive to the mesh quality (especially close to the wall) for the quiescent flow initial conditions than for the established flow initial conditions. In this study, all numerical results were obtained by using the established flow initial condition.

For one set of Bingham fluid simulations, the Bingham number is increased from $B n=0$ until the pureconduction solution is obtained for a large value of $B n$. Reducing $B n$ from this condition to $B n=0$ in other set of simulations recovers the same mean Nusselt number and flow pattern. Thus, no significant hysteresis was observed in the current analysis.

\section{SCALING ANALYSIS}

A scaling analysis was carried out to elucidate the expected influences of Reynolds, Richardson, Rayleigh, Prandtl and Bingham numbers on the mean Nusselt number. The wall heat flux can be scaled as:

$$
q \sim k \frac{\Delta T}{\delta_{t h}} \sim h \Delta T
$$

Using Eq. (19), the Nusselt number can be scaled as:

$$
N u \sim \frac{h R}{k} \sim \frac{R}{\delta_{t h}}
$$

Or:

$$
N u \sim \frac{R}{\delta} f_{1}(R e, R i, R a, P r, B n)
$$

where $f_{1}$ is a function of $R e, R i, R a, P r$ and $B n$, which accounts for the ratio of hydrodynamic to thermal boundary layer thicknesses (i.e. $\delta / \delta_{t h} \sim f_{1}(R e, R i, R a, P r, B n)$ ). 
In order to estimate the hydrodynamic boundary thickness $\delta$, the equality between the order of magnitudes of inertial and viscous forces in the radial direction can be considered:

$$
\rho \frac{V^{2}}{R} \sim \frac{\tau}{\delta}
$$

For Bingham fluids, the shear stress can be scaled as $\tau \sim \tau_{y}+\mu(U / \delta)$ and thus Eq. (21) can be rewritten as:

$$
\rho \frac{V^{2}}{R} \sim\left(\tau_{y}+\mu \frac{U}{\delta}\right) \frac{1}{\delta}
$$

Using Eq. (22), the hydrodynamic boundary thickness $\delta$ can be estimated as:

$$
\delta \sim \frac{1}{2} \frac{\tau_{y} R}{\rho V^{2}}+\frac{1}{2} \frac{R}{\rho V^{2}} \sqrt{\tau_{y}^{2}+\frac{4 \rho \mu U V^{2}}{R}}
$$

Here, $U$ and $V$ are the characteristic velocity scales in the radial and tangential directions, respectively, and they can be estimated as shown in Table 3 for different flow conditions. Eq. (23) can be rewritten by using the velocity scale $U \sim a(\Omega R)+b(\sqrt{g \beta \Delta T R})$ as follows:

$$
\frac{\delta}{R} \sim \frac{B n}{2 R e}+\frac{1}{2 R e} \sqrt{B n^{2}+4\left[a R e+b\left(\frac{R a}{P r}\right)^{1 / 2}\right]}
$$

where the parameters $a=e^{-\theta R i}$ and $b=1-e^{-\theta R i}$ (with $\theta$ being a parameter) ensure that $U \sim(\Omega R)$ for small values of $R i$ (i.e. for forced convection) whereas one obtains $U \sim \sqrt{g \beta \Delta T R}$ for large values of $R i$ (i.e. for natural convection). According to $R i$, Eq. (24) gives different results. For example, for fully forced convection, which indicates $R i=0$, Eq. (24) yields:

$$
\frac{\delta}{R} \sim \frac{B n}{2 R e}+\frac{1}{2 R e} \sqrt{B n^{2}+4 R e}
$$

For $R i \gg 1$ (when natural convection dominates the flow) one obtains:

$$
\frac{\delta}{R} \sim \frac{B n}{2 R e}+\frac{1}{2 R e} \sqrt{B n^{2}+4\left(\frac{R a}{P r}\right)^{1 / 2}}
$$


Equation (25b) indicates that the effects of rotation do not disappear even for $R i \gg 1$ cases. It will be demonstrated later in this paper that the mean Nusselt number asymptotically approaches unity where the fully conduction-driven transport occurs after a sudden drop in the mean Nusselt number with increasing Bingham number. This implies that the advective transport could be sustained in this configuration up to a higher value of nominal Bingham number than the value obtained for pure natural convection (i.e.Ri 》 1) [26]. Substituting Eq. (24) into Eq. (20b) leads to the following scaling estimate for the mean Nusselt number:

$$
\overline{N u} \sim \frac{2 R e}{B n+\sqrt{B n^{2}+4\left[a R e+b\left(\frac{R a}{P r}\right)^{\frac{1}{2}}\right]}} f_{1}(R e, R i, P r, B n)
$$

Equation (26) can also be rewritten based on Richardson number using the relation between $R i$ and $R a$ (i.e. $\left.R a / \operatorname{Pr}=R i R e^{2}\right)$ :

$$
\overline{N u} \sim \frac{2 R e}{B n+\sqrt{B n^{2}+4 R e\left[a+b R i^{\frac{1}{2}}\right]}} f_{1}(R e, R i, \operatorname{Pr}, B n)
$$

According to Eq. (27), $\overline{N u}$ is expected to decrease with increasing $B n$ for a given set of values of $R e$ and $R i$. Similarly, for a given set of values of $B n$ and $R e$, the mean Nusselt number decreases with increasing $R i$. On the other hand, $\overline{N u}$ increases with increasing $R e$ for a given set of values of $R i$ and $B n$. Finally, Eq. (26) offers important physical insight into the influences of $R e, R i$, and $B n$ on the mean Nusselt number $\overline{N u}$ for both Newtonian and Bingham fluid cases. The important results of this scaling analysis are summarised in Table 4.

\section{RESULTS \& DISCUSSIONS}

According to Buckingham's pi theorem it is possible to show that the mean Nusselt number $\overline{N u}$ can be expressed as $\overline{N u}=f_{2}(B n, R e, R i, P r)$. Similarly, the dimensional analysis indicates that the torque coefficient, $C_{T}$, for this configuration can be taken as: $C_{T}=f_{3}(B n, R e, R i, \operatorname{Pr})$. Thus, it is instructive to 
analyse the effects of $B n, R e, R i$ and $\operatorname{Pr}$ on $\overline{N u}$ and $C_{T}$, which are discussed in sub-Sections 4.1 and 4.2, respectively, based on the findings of the numerical simulations.

\subsection{Effects of $B n, R e, R i$ and $P r$ on the mean Nusselt number $\overline{N u}$}

\subsubsection{Influence of Bingham number}

The variation of the mean Nusselt number $\overline{N u}$ with Bingham number $B n$ is shown in Fig. 3 for $R e=500$ and $R i=0$ at $\operatorname{Pr}=500$ (which represents a realistic value of $\operatorname{Pr}$ for aqueous Carbopol solution [27], which is often taken to be a model yield stress fluid for experimental investigations). It is evident from Fig. 3 that $\overline{N u}$ decreases sharply with increasing $B n$, but subsequently $\overline{N u}$ approaches asymptotically to a value of unity, which is indicative of conduction-driven transport. It is also shown in Fig. 3 that the isotherms are curved and not parallel to horizontal walls, especially in the vicinity of the rotating heated top cover, even for $\overline{N u}=1.001$, which indicates that the effects of advective transport sustain even for small values of mean Nusselt number. Therefore, in this study, the criterion for the fully conductiondominated regime is taken to be $\overline{N u}=1.0001$ because the isotherms for this condition remain parallel to the horizontal walls as dictated by a pure-conduction solution. The variation of $\overline{N u}$ with $B n$ shown in Fig. 3 is consistent with the scaling estimate given by Eq. 27, which suggests that $\overline{N u}$ is expected to decrease with increasing $B n$. In order to elucidate the behaviour of $\overline{N u}$ at high values of Bingham number, the variation of $\overline{N u}$ with $B n$ is rearranged using a logarithmic scale, as demonstrated in Fig. 4, where the variations of $\overline{N u}$ with $B n^{*}=\log \left(B n^{2}+1\right)$ are shown for nominal values of Reynolds number $R e=$ 500, 1000 and 2000 and Richardson number $R i=0$ and 1 at $P r=500$. It can be seen from Fig. 4 that viscous resistance strengthens in comparison to buoyancy and inertial forces with increasing $B n$ for both forced (i.e.Ri $=0)$ and mixed (i.e. $R i=1)$ convection cases. The fluid flow becomes so weak for large values of $B n$ that it imparts no influence on thermal transport and heat transfer takes place principally owing to thermal conduction. 
The Bingham number at which the mean Nusselt number approaches 1.0001 is referred to $B n_{\max }$ in this paper. Using Eq. (27), one can estimate $B n_{\max }$ as:

$$
B n_{\text {max }} \sim R e f_{1}-\left(a+b R i^{0.5}\right) / f_{1}
$$

Thus, $B n_{\max }$ is a threshold Bingham number that indicates that the advective transport remains important (unimportant) for $B n<B n_{\max }\left(B n>B n_{\max }\right)$. Eq. (28) suggests that the advective transport can be sustained for larger value of Bingham number for higher values of Reynolds number, and this behaviour can be confirmed by the numerical results presented in Fig. 4. The effects of advective transport strengthen with increasing $R e$. As a result of the stronger advective transport, the Bingham number at which $\overline{N u}$ approaches to 1.0001 (i.e. $B n=B n_{\max }$ ) increases with increasing $R e$. This behaviour can further be seen by comparing the contours of stream function and non-dimensional temperature in Fig. 5 for $R i=$ 0 and 1, for different values of $B n$ at $R e=2000$ and $P r=500$. The grey regions on the streamline plots in Fig. 5 indicate the Apparently Unyielded Regions (AURs) (regions where $|\tau| \leq \tau_{y}$ [28]). According to biviscosity regularisation, the unyielded zones are replaced by the regions of high viscosity—regions of extremely slowly moving fluid (Mitsoulis and Zisis [28] called them "apparently unyielded regions (AUR)"). It is worth noting that fluid flow does not stop in a true sense in the context of biviscosity regularisation. In the unyielded regions the flow becomes so weak (i.e. the magnitudes of velocity and stream function) that these regions can effectively be considered as the stagnant zones. The velocity magnitudes in these zones are so small that they do not impart any influence on thermal transport. An idea about the locations of these unyielded zones can be obtained from the AURs. However, it is important to note that AURs are not really "unyielded" in the true sense, as pointed out by Mitsoulis and Zisis [28]. The shapes and sizes of AURs are dependent on the choice of $\mu_{y i e l d}$, but the qualitative and quantitative distributions of streamlines and isotherms remain independent of the value of $\mu_{\text {yield }}$ (at least for $\mu_{y i e l d} /$ $\mu=10^{8}$ ). Thus the precise shape and size of the AURs do not have any major influence on the $\overline{N u}$ in this analysis. Fig. 5 shows a large region of almost uniform temperature with low temperature regions concentrated close to the axis and at the bottom of the container for the pure forced convection (i.e. $R i=$ 
0) case for Newtonian (i.e. $B n=0$ ) fluids, whereas the low temperature region is concentrated only at the bottom of the container for the mixed convection (i.e. $R i=1)$ case. The large region of almost uniform temperature is indicative of a high heat transfer rate in this case. The region of low temperature moves to the bottom of the container with increasing $B n$ and the isotherms become increasingly parallel to top (or bottom) cover of the container with a gradual increase in temperature from the bottom to top of the container with large thermal boundary layer at the top. Additionally the sizes of the AURs expand with increasing $B n$ and almost the whole domain becomes unyielded for large values of $B n$, which indicates that the flow practically stops under this condition. This behaviour is indicative of strengthening of conduction-driven transport with increasing $B n$, and the heat transfer takes place principally owing to conduction, when $B n$ reaches $B n_{\max }$.

\subsubsection{Influence of Reynolds number}

The variation of the mean Nusselt number $\overline{N u}$ with nominal Reynolds number $R e$ for $R i=0$ (i.e. purely forced convection) and 1 (i.e. mixed convection) at $P r=500$ is shown in Fig. 6. It can be seen from Fig. 6 that $\overline{N u}$ increases with increasing $R e$ for both $R i=0$ and 1.0 in the case of both Newtonian and Bingham fluids, which is consistent with the scaling estimate of the mean Nusselt number (see Eq. (27)). Fig. 6 also shows that the values of $\overline{N u}$ for Bingham fluids are smaller than those obtained in the case of Newtonian fluids with the same nominal Reynolds number, Re. This behaviour is also in agreement with the scaling estimate of Nusselt number given by Eq. (27), which predicts a smaller value of $\overline{N u}$ for Bingham fluids for a given value of $R e$. In order to understand the effects of Reynolds number on thermal transport in this configuration, the distributions of non-dimensional temperature $\theta$ and normalised swirl velocity component $V_{\theta} / V_{\theta_{\max }}$ along the vertical mid-plane $(r / R=0.5)$ for both Newtonian $(i . e . B n=0)$ and Bingham fluids (for $B n=1$ and 10) are presented in Fig. 7 for $R i=0.1$ for different values of Reynolds number. It can be observed from Fig. 7 that the thermal boundary layer thickness on the bottom cover decreases with increasing Reynolds number for both Newtonian and Bingham cases. This is also 
consistent with the scaling estimate of $\delta_{t h}$ given by Eq. (24). This thinning of the boundary layer with increasing $R e$ eventually leads to an increase in the Nusselt number $\left(\overline{N u} \sim h R / k \sim R / \delta_{t h}\right)$, as shown in Fig. 6. An increase in Nusselt number with increasing Reynolds number bears the signature of strengthening of advective transport. This can be substantiated from Fig. 7, which shows non-negligible values of $V_{\theta} /$ $V_{\theta_{\text {max }}}$ over a large region for high values of $R e$. It is worth noting that $V_{\theta_{\max }}$ increases with increasing $R e$ for a given value of $P r$. Here, $V_{\theta}$ is normalised by $V_{\theta_{\max }}$ to demonstrate the effects of $R e$ on the hydrodynamic boundary layer thickness. Figs. 6 and 7 indicate that the influences of Reynolds number $R e$ on both the mean Nusselt number $\overline{N u}$ and the magnitude of swirling velocity $V_{\theta}$ weaken with increasing $B n$. This result is an artefact of how the nominal Reynolds number $R e$ is defined in the present analysis (see Eq. (10)). In the case of Bingham fluids, the use of an effective viscosity instead of a constant plastic viscosity in the definition of Reynolds number would have been more appropriate. The "effective" viscosity can be estimated as:

$$
\mu_{e f f} \sim \tau_{y} / \dot{\gamma}+\mu
$$

which can be scaled as:

$$
\mu_{e f f} \sim \tau_{y} \delta / U+\mu
$$

Using Eq. (24) in Eq. (30) and using the velocity scales $U \sim a(\Omega R)+b(\sqrt{g \beta \Delta T R})$ and $V \sim \Omega R$ yields:

$$
\mu_{e f f} \sim \frac{\tau_{y} R}{\mathrm{a}(\Omega R)+b \sqrt{g \beta \Delta T R}}\left(\frac{B n}{2 R e}+\frac{1}{2 R e} \sqrt{B n^{2}+4\left[a R e+b R e R i^{1 / 2}\right]}\right)+\mu
$$

Based on Eq. (31), an effective Reynolds number $R e_{\text {eff }}$ can be defined as:

$$
R e_{e f f}=\frac{\rho \Omega R^{2}}{\mu_{e f f}}=\frac{R e\left(a+b R i^{1 / 2}\right)}{\left(a+b R i^{\frac{1}{2}}\right)+\left(\frac{B n^{2}}{2 R e}+\frac{B n}{2 R e} \sqrt{B n^{2}+4\left[a R e+b R e R i^{1 / 2}\right]}\right)}
$$

Eq. (32) suggests that the effective Reynolds number $R e_{e f f}$ decreases with increasing $B n$ for a given value of $R i$ which is consistent with the observation in Figs. 6 and 7.

\subsubsection{Influence of Richardson number}


The variation of $\overline{N u}$ with $R i$ is presented in Fig. 8 for different Bingham number $B n$ values for $R e=1000$ at $P r=500$. It can be seen from Fig. 8 that $\overline{N u}$ shows a decreasing trend with increasing $R i$ for Newtonian fluid (i.e. $B n=0)$ and also for Bingham fluid cases with small values of Bingham number, whereas $\overline{N u}$ remains insensitive to the changes in $R i$ in the case of large Bingham number. According to the scaling estimation given by Eq. (27), the mean Nusselt number is expected to decrease with increasing $R i$ for small values of $B n$. For large values of $B n$ (where $\left.B n^{2} \gg R e R i^{0.5}\right) \overline{N u}$ is principally determined by $R e$ and $B n$, and thus the mean Nusselt number does not show any appreciable $R i$ dependence. The observed $R i$ dependence of $\overline{N u}$ can further be explained from the distributions of the non-dimensional temperature $\theta$ and normalised swirl velocity component $V_{\theta} / V_{\theta \max }$ along the vertical mid-plane, which are presented in Fig. 9 for different $B n$ values at $R e=1000$ and $P r=500$. It can be seen from Fig. 9 that the distributions of both temperature and swirling velocity component are affected by $R i$ for small values of $B n$ where the thermal boundary layer thickens with increasing $R i$, which leads to a decrease in the mean Nusselt number, as shown in Fig 9. Furthermore, $R i$ does not have any influence on the non-dimensional temperature $\theta$ and normalised swirl velocity component $V_{\theta} / V_{\theta \max }$ for large values of Bingham number $B n$ and thus $\overline{N u}$ does not get affected by Richardson number $R i$, as shown in Fig. 8 .

For mixed convection, the relative strengths of inertial, buoyancy and viscous forces determine the flow behaviour. For $R i=0$, which corresponds to purely forced convection, the flow is governed by the inertial and viscous forces. The influence of buoyancy force starts to strengthen with an increase in $R i$, and therefore the competition between buoyancy force and viscous forces starts to become increasingly important with increasing $R i$. However, this situation where the top cover is hotter than the bottom one represents a stable configuration where the lighter hot fluid sits on top of heavier cold fluid so the effects of natural convection only remain significant close to the heated top cover in the container. This can also be observed from the variation of the normalised swirling velocity component along to the vertical midplane in Fig. 9, which shows that the magnitude of $V_{\theta} / V_{\theta \max }$ assumes higher values with increasing $R i$ 
only near the hot (top) cover in the case of small Bingham numbers. However, the magnitude of $V_{\theta} / V_{\theta \max }$ decreases with increasing $R i$ towards the bottom of the container. As a result of this, advective transport weakens and the mean Nusselt number decreases with increasing $R i$ for small values of $B n$. For large values of $B n$, the region of non-negligible value of $V_{\theta} / V_{\theta \max }$ remains confined to the vicinity of the rotating top cover and thus the strength of advective transport and the mean Nusselt number are not significantly affected by $R i$.

\subsubsection{Influence of Prandtl number}

The variations of $\overline{N u}$ with $\operatorname{Pr}$ for Newtonian $(B n=0)$ and Bingham fluids $(B n=1$ and 10) for both $R i=0$ (pure forced convection) and $R i=1$ (mixed convection) cases are shown in Fig. 10, which indicates that $\overline{N u}$ increases with increasing $\operatorname{Pr}$. Fig. 10 shows that $\operatorname{Pr}$ has an important influence on $\overline{N u}$ for small values of $B n$ for both $R i=0$ and 1.0 cases, whereas $\overline{N u}$ remains insensitive to the variation of $\operatorname{Pr}$ for high values of $B n$. In order to gain insight into this behaviour the distributions of $\theta$ and $V_{\theta}$ along the vertical mid-plane are shown in Fig. 11 for $R i=1.0$. It is apparent from Fig. 11 that the thermal boundary layer thickness decreases with increasing $\operatorname{Pr}$ for small values of Bingham number, which in turn acts to increase the mean Nusselt number $\overline{N u} \sim R / \delta_{t h}$. Furthermore, the magnitude of the non-dimensional swirling velocity component rises with increasing $\operatorname{Pr}$ for all Bingham number cases. This indicates that the advective transport strengthens with increasing $P r$, which is consistent with previous findings [8]. This behaviour is observed all along the vertical mid-plane for small values of $B n$, whereas it appears only in the vicinity of the heated top cover for large $B n$ values. Therefore, $\overline{N u}$ is less sensitive to the variation of $\operatorname{Pr}$ for large values of $B n$ as shown in Fig. 10.

\subsubsection{Parameterisation of $B n_{\max }$}

The variations of $B n_{\max }$ with $R e$ for different values of $\operatorname{Pr}$ and $R i$ are presented in Fig. 12 and the values of $B n_{\max }$ are presented in Table 5. It can be seen from Fig. 12 that $B n_{\max }$ increases with increasing $R e$ 
and $\operatorname{Pr}$ owing to the strengthening of advective transport (which has already been discussed in detail in the previous sub-sections). However, as mentioned in Section 4.1.3, the influence of $R i$ on $\overline{N u}$ disappears for large values of $R i$ so $B n_{\max }$ is not influenced by $R i$. Here, $B n_{\max }$ has been parameterised by fitting the simulation results in the following manner:

$$
B n_{\text {max }}=0.04 \operatorname{Re}^{1.08} \operatorname{Pr}^{0.57}
$$

It can be seen from Fig. 12 that Eq. (33) satisfactorily captures the numerical predictions for $B n_{\max }$. In addition to this, it needs to be mentioned that the results of the numerical simulation and Eq. (33) are to be treated with caution because $\overline{N u}=1.0001$ is not sufficient for identifying the critical condition under which flow stops in the enclosure. Furthermore, for bi-viscosity regularisation, the flow does not stop in a true sense for $B n \geq B n_{\max }$, and under this condition the flow is present but it becomes extremely weak to impart any significant influence on the thermal transport. Thus, the identification of the critical condition in terms of Bingham number for which flow stops is not important from the perspective of heat transfer analysis conducted here.

\subsubsection{The mean Nusselt number correlation}

In the case of Newtonian fluids, the following correlation function is proposed for the mean Nusselt number $\overline{N u}$ for $0 \leq R i \leq 1,500 \leq R e \leq 3000$ and $10 \leq \operatorname{Pr} \leq 500$ :

$$
\overline{N u}_{B n=0}=1+k_{0} R e^{m_{0}}
$$

where $k_{\mathrm{o}}$ and $m_{\mathrm{o}}$ are the correlation parameters in which are listed in Table 6. The prediction of Eq. (34) is compared with $\overline{N u}_{B n=0}$ obtained from simulation data in Fig. 13, which shows a good agreement between the correlation predictions and simulation results for the range of $R i, R e$ and $\operatorname{Pr}$ considered here.

Based on the scaling relation given by Eq. (27), a correlation for the mean Nusselt number for Bingham fluids is also proposed here for $0 \leq R i \leq 1,500 \leq R e \leq 3000$ and $10 \leq \operatorname{Pr} \leq 500$ : 


$$
\overline{N u}=\operatorname{Max}\left[1, \frac{\overline{N u}_{B n=0}}{\left(\frac{B n}{A}+\sqrt{\left.\left(\frac{B n}{A}\right)^{2}+1\right)} D\right.}\right]
$$

where $A=2 \sqrt{a R e+b\left(\operatorname{RiRe}^{2}\right)^{1 / 2}}$ when $a=e^{-R i}$ and $b=1-e^{-R i}$. Here, $D$ is expressed as: $D=1+$ $k_{1} B n^{m_{1}}$ where $k_{1}$ and $m_{1}$ are the correlation parameters, which are listed in Table 7. For $B n=0$, Eq. (35) reduces to the mean Nusselt number correlation for Newtonian fluids (i.e. Eq. (34)). By contrast, for $B n \geq$ $B n_{\max }$, the mean Nusselt number $\overline{N u}$ approaches unity owing to conduction-driven transport. The predictions of Eq. (35) are compared to the numerical data in Fig. 14 for $\operatorname{Pr}=10,100$ and 500, which demonstrates that the correlation given by Eq. (35) satisfactorily captures the qualitative variations of $\overline{N u}$ with $B n$ for the range of $R i, R e$ and $\operatorname{Pr}$ analysed in this study. The correlation given by Eq. (35) has been found to approximate the numerical results at the level of average error of $10 \%$ and $13 \%$ for $\operatorname{Pr}=10,100$ and $\operatorname{Pr}=500$, respectively. Although Eq. (35) slightly underestimates the magnitude of $\overline{N u}$ for $R e=500$, the agreement between the prediction of Eq. (35) with computational results improves with increasing $R e$.

\subsection{Effects of $B n, R e, R i$ and $P r$ on torque coefficient $C_{T}$}

The variation of the torque coefficient, $C_{T}$, with Bingham number, $B n$, for different values of Reynolds numbers for $R i=0$ and $R i=1$ at $P r=500$ are shown in Fig. 15a. It can be seen from Fig. 15a that $C_{T}$ increases with increasing $B n$ as the viscous resistance increases with increasing Bingham number. This indicates that more power is needed to produce a swirl flow inside the container for higher values of $B n$. This result can be confirmed by a scaling analysis for the torque coefficient $C_{T}$. Using shear stress scaling $\left(\tau \sim \tau_{y}+\mu(V / \delta)\right)$ for Bingham fluids, Eq. (16b) can be scaled as follows:

$$
C_{T} \sim \frac{\left(\tau_{y}+\frac{\mu V}{\delta}\right) R^{3}}{\rho \Omega^{2} R^{5}}
$$

Eq. (36) can be rewritten by using the velocity scale $V \sim \Omega R$ as:

$$
C_{T} \sim \frac{1}{R e}\left(B n+\frac{R}{\delta}\right)
$$


Eq. (37) can be recast using Eq. (24) as follows:

$$
C_{T} \sim \frac{B n}{R e}+\frac{2}{B n+\sqrt{B n^{2}+4\left(a R e+b R e R i^{1 / 2}\right)}}
$$

Eq. (38) suggests that $C_{T}$ increases with increasing $B n$ more rapidly for smaller values of $R e$.

The variation of torque coefficient $C_{T}$ with Reynolds number $R e$ for both Newtonian and Bingham fluids is shown in Fig. $15 \mathrm{~b}$ for $R i=0$ and 1 at $\operatorname{Pr}=500$. The torque coefficient $C_{T}$ decreases with increasing $R e$ for a given set of values of $B n$ and $\operatorname{Pr}$ for both $R i=0$ and 1 . This trend is also consistent with the scaling estimation given by Eq. (38). The viscous resistance weakens in comparison to inertial and buoyancy forces with increasing $R e$ and this gives rise to a decrease in $C_{T}$. This implies that the power needed to produce a swirling flow in the container decreases with increasing Reynolds number. An increase in $B n$ leads to a decrease in $R e_{e f f}$, which gives rise to an increase in $C_{T}$ with increasing $B n$.

The variation of torque coefficient $C_{T}$ with Richardson number for different Bingham numbers at $R e=$ 1000 and $\operatorname{Pr}=500$ is shown Fig. 15c. The figure shows that $C_{T}$ slightly decreases with increasing $R i$ values for small $B n$ values, whereas it does not change with $R i$ for large $B n$ values. This is also consistent with the scaling estimate given by Eq. (38), which indicates a decrease in $C_{T}$ with increasing $R i$. Strengthening of natural convection (i.e. buoyancy force) with increasing $R i$ does not affect the whole flow field but only acts to reduce the velocity gradient in the vicinity of the heated top cover for small values of $B n$ (see Fig. 9). This gives rise to a marginal decrease in $C_{T}$ with increasing $R i$ for small values of $B n$. However, for large values of $B n$, the velocity gradient near the top (hot) cover remains independent of $R i$ and therefore the shear stress and the torque coefficient $C_{T}$ are not influenced by $R i$.

The variations of $C_{T}$ with $\operatorname{Pr}$ for different values of $B n$ in the case of pure forced convection (i.e. $R i=0$ ) and mixed convection $(R i=1)$ are shown in Fig. 15d. For fully forced convection (i.e. $R i=0)$ case, $P r$ does not have a significant influence on $C_{T}$, which is consistent with the scaling estimation given by Eq. 
(38) for $R i=0$ (i.e. $C_{T} \sim B n / R e+2 / B n+\sqrt{B n^{2}+4 a R e}$ ). The difference between hydrodynamic to thermal boundary layer thickness increases with increasing $P r$. Thus, the interaction between inertial and viscous forces takes place outside the thermal boundary layer within the hydrodynamic boundary layer for $\operatorname{Pr} \gg 1$, and as a result the viscous resistance that affects the magnitude of $C_{T}$ remain largely independent of heat transfer within the thermal boundary layer in the case of forced convection (i.e. $R i=0)$. However, in the case of mixed convection (i.e. $R i=1$ ) the buoyancy force imparts a significant influence on the flow over the length scale comparable to the thermal boundary layer for small values of $\operatorname{Pr}$ and affects $C_{T}$, but this effect levels out as the separation between hydrodynamic and thermal boundary layer thicknesses increases with increasing $\operatorname{Pr}$ (Fig. 15d). As discussed earlier, natural convection does not become strong enough to overcome the viscous effects for the whole of the container for the parameter range considered here. Thus, the effects of natural convection remain strong only in the vicinity of the heated top wall and so $C_{T}$ gets marginally affected by the variation of $P r$. The flow resistance increases with increasing $B n$, which gives rise to an increase in $C_{T}$ with increasing $B n$. This is also consistent with the scaling estimation given by Eq. (38), which suggests an increase in $C_{T}$ with increasing $B n$.

\section{CONCLUSIONS}

The influences of Reynolds, Prandtl, Richardson and Bingham numbers on the mean Nusselt number and torque coefficient for steady laminar mixed convection of yield stress fluids obeying the Bingham model in cylindrical enclosures with a heated rotating top cover have been analysed based on numerical simulations. The mean Nusselt number $\overline{N u}$ has been found to increase with increasing Reynolds number for both Newtonian and Bingham fluids owing to strengthening of advective transport. Moreover, $\overline{N u}$ for Bingham fluids assumes smaller values than that obtained in the case of Newtonian fluids with same nominal values of Reynolds number, $R e$, owing to the weakening of advective transport as a result of the additional flow resistance arising from yield stress. It has been found that $\overline{N u}$ decreases sharply with increasing $B n$, but subsequently $\overline{N u}$ asymptotically approaches a value of unity, which is indicative of 
conduction-driven transport. The mean Nusselt number, $\overline{N u}$, shows a decreasing trend with increasing $R i$ for Newtonian fluids (i.e. $B n=0$ ) and also for Bingham fluids for small values of Bingham number, whereas $\overline{N u}$ remains insensitive to $R i$ for large values of Bingham number. It has also been observed that $\operatorname{Pr}$ has an important influence on $\overline{N u}$ for small values of $B n$, whereas $\overline{N u}$ remains insensitive to the variation of $\operatorname{Pr}$ for large values of $B n$. The mean Nusselt number $\overline{N u}$ has been found to increase with increasing $\operatorname{Pr}$ for Newtonian fluids.

The torque coefficient, $C_{T}$, has been found to decrease with increasing $R e$ for a given set of values of $R i$, $B n$ and $P r$. Moreover, the torque coefficient, $C_{T}$, has been found to increase with increasing $B n$ as the viscous resistance increases with increasing Bingham number. In addition, the torque coefficient $C_{T}$ decreases slightly with increasing $R i$ for small values of $B n$, whereas $C_{T}$ does not change with $R i$ for large $B n$ values. For fully forced convection (i.e. $R i=0$ ) case, $\operatorname{Pr}$ does not have any significant influence on the $C_{T}$. However, in the case of mixed convection $(e . g . R i=1) C_{T}$ exhibits weak Prandtl number dependence for small values of $\operatorname{Pr}$ but this dependence disappears as the separation between hydrodynamic and thermal boundary layer thicknesses increases with increasing $\operatorname{Pr}$.

Mixed convection of yield stress fluids within a cylindrical enclosure with a heated top cover has applications in the pharmaceutical and food-processing industries (e.g. ice cream preparation). Thus, the physical insights and the correlations offered by the present analysis in terms of the statistical behaviours of the mean Nusselt number and torque coefficient for mixed convection of Bingham fluids will be useful to optimise the performance of heat transfer and mixing in the aforementioned practical applications.

\section{ACKNOWLEDGEMENTS}

This study was supported by the Newton Research Collaboration Programme and is hereby gratefully acknowledged. 


\section{REFERENCES}

[1] H. U. Vogel, Experimentelle Ergebnisse über die laminare Strömung in einem zylindrischen Gahause mit darin rotieren-der Scheibe. MPI Bericht 6, (1968).

[2] H. U. Vogel, Rückströmungsblasen in Drallsströmungen. Festschrift 50 Jahre Max-Planck-Institut für Strömungsforschung 1925-1975, (1975).

[3] B. Ronnenberg, Ein selbstjustierendes 3-Komponenten-Laserdoppleranemometer nach dem Vergleichsstrahlverfahren, angewandt für Untersuchungen in einer stationaren sylinder-symmetrischen Drehströmung mit einem Rückstromgebiet. MPI Bericht 20, (1977).

[4] M. Bertela, F. Gori, Laminer flow in a cylindrical container with a rotating cover, J. Fluids Eng. 104 (1982) 31-39.

[5]M.P. Escudier, Observations of the flow produced in a cylindrical container by rotating endwall, Experiments in Fluids 2 (1984) 189-196.

[6] H.J. Lugt, H.J. Haussling, Axisymmetric vortex breakdown in rotating fluid within a container, Trans. ASME J. Appl. Mech. 49 (1982) 921-923.

[7] J.M. Lopez, Axisymmetric vortex breakdown: Part1. Confined swirling flow, J. Fluid Mech. $221(1990) 533-552$.

[8] C.H. Lee, J.M. Hyun, Flow of a stratified fluid in a cylinder with a rotating lid, Int. J. Heat and Fluid Flow 20 (1999) 26-33.

[9] R. Iwatsu, Flow pattern and heat transfer of swirling flows in cylindrical container with rotating top and stable temperature gradient, Int. J. Heat and Mass Transfer 47 (2004) 2755-2767.

[10] M. P. Escuider, J. O'Leary, R.J. Poole, Flow produced in a conical container by a rotating end wall, Int. J. of Heat and Fluid Flow 28 (2007) 1418-1428.

[11] A. Acrivos, M. J. Shah, E. E. Petersen, On the flow of a non-Newtonian liquid on a rotating disk, J. Appl. Phys. 31(6) (1960) 963-968.

[12] L. Elliott, Elastico-viscous flow near a rotating disk, Phys. Fluids 14(6) (1971) 1086-1090. 
[13] D. D. Kale, R. A. Mashelkar, J. Ulbrecht, Rotational viscoelastic laminar boundary layer flow around a rotating disc, Rheol. Acta 14(7) (1975) 631-640.

[14] M.P. Escudier, L. M. Cullen, Flow of a shear-thinning liquid in a cylindricalcontainer with a rotating end wall, Experimental Thermal and Fluid Science 12 (1996) 381-387.

[15] J. R. Stokes and D. V. Boger, Mixing of viscous polymer liquids, Phys. Fluids 12(6) (2000) 14111416.

[16] B. Traore, C. Castelain and T. Burghelea, Efficient heat transfer in a regime of elastic turbulence, Journal of Non-Newtonian Fluid Mechanics 223 (2015) 62-76.

[17] G. Böhme, L. Rubart, M. Stenger, Vortex breakdown in shear-thinning liquids: experiment and numerical simulations, J. Non-Newtonian Fluid Mech. 45 (1992) 1-20.

[18] A. A. Rashaida, D. J. Bergstrom, and R. J. Sumner, Mass transfer from a rotating disk to a Bingham fluid, Trans. ASME J. Appl. Mech. (2006) 73 108-111.

[19] A. Ahmadpour and K. Sadeghy, Swirling flow of Bingham fluids above a rotating disk: An exact solution, J. Non-Newtonian Fluid Mech. 197 (2013) 41-47.

[20] A. Guha, S. Sengupta, Analysis of von Kármán’s swirling flow on a rotating disc in Bingham fluids, Phys. Fluiids 28 (2016) 013601.

[21] H. A. Barnes, The yield stress - a review or ' $\pi \alpha v \tau \alpha \rho \varepsilon \iota$ ' - everything flows? J. Non-Newt. Fluid Mech. 81 (1999) 133-178.

[22] E.J. O’Donovan, R.I. Tanner, Numerical study of the Bingham squeeze film problem, J. NonNewtonian Fluid Mech. 15 (1984) 75-83.

[23] R.J. Poole, R.P. Chhabra, Development length requirements for fully-developed laminar pipe flow of yield stress fluids, ASME J. Fluids Eng. 132(3) (2010) 034501.

[24] S. V. Patankar, Numerical Heat Transfer and Fluid Flow, Hemisphere, Washington, D.C, 1980.

[25] O. Turan, N. Chakraborty, R. J. Poole, Laminar natural convection of Bingham fluids in a square enclosure with differentially heated side walls, J. Non-Newtonian Fluid Mech. 165 (2010), 901-913. 
[26] S. Yigit, R. J. Poole, N. Chakraborty, Effects of aspect ratio on laminar Rayleigh-Bénard convection of power-law fluids in rectangular enclosures: A numerical investigation, Int. J. Heat and Mass Trans. 91 (2015) 1292-1307.

[27] Z. Kebiche, C. Castelain, T. Burghelea, Experimental investigation of the Rayleigh-Bénard convection in a yield stress fluid, J. Non-Newt. Fluid Mech. 203 (2014) 9-23.

[28] E. Mitsoulis, T. Zisis, Flow of Bingham plastics in a lid-driven square cavity, J. Non-Newtonian Fluid Mech. 101 (2001) 173-180. 
Table 1. The normalised minimum grid spacing $\Delta_{\text {min,cell }} / R$ and grid expansion ratio $r_{e}$

\begin{tabular}{lccc}
\hline & $\mathrm{M} 1(50 \times 50)$ & $\mathrm{M} 2(100 \times 100)$ & $\mathrm{M} 3(200 \times 200)$ \\
\hline$\Delta_{\text {min,cell }} / R$ & $4.60 \times 10^{-3}$ & $2.30 \times 10^{-3}$ & $1.15 \times 10^{-3}$ \\
$r_{e}$ & 1.0287 & 1.0141 & 1.0070 \\
\hline
\end{tabular}


Table 2. The numerical uncertainty for the mean Nusselt number $\overline{N u}$ and maximum non-dimensional stream function $\Psi_{\max }\left(=\psi_{\max } / \alpha\right)$ for Newtonian and Bingham fluids for $R i=0.1$ and $R e=1000$ at $\operatorname{Pr}=$ 500.

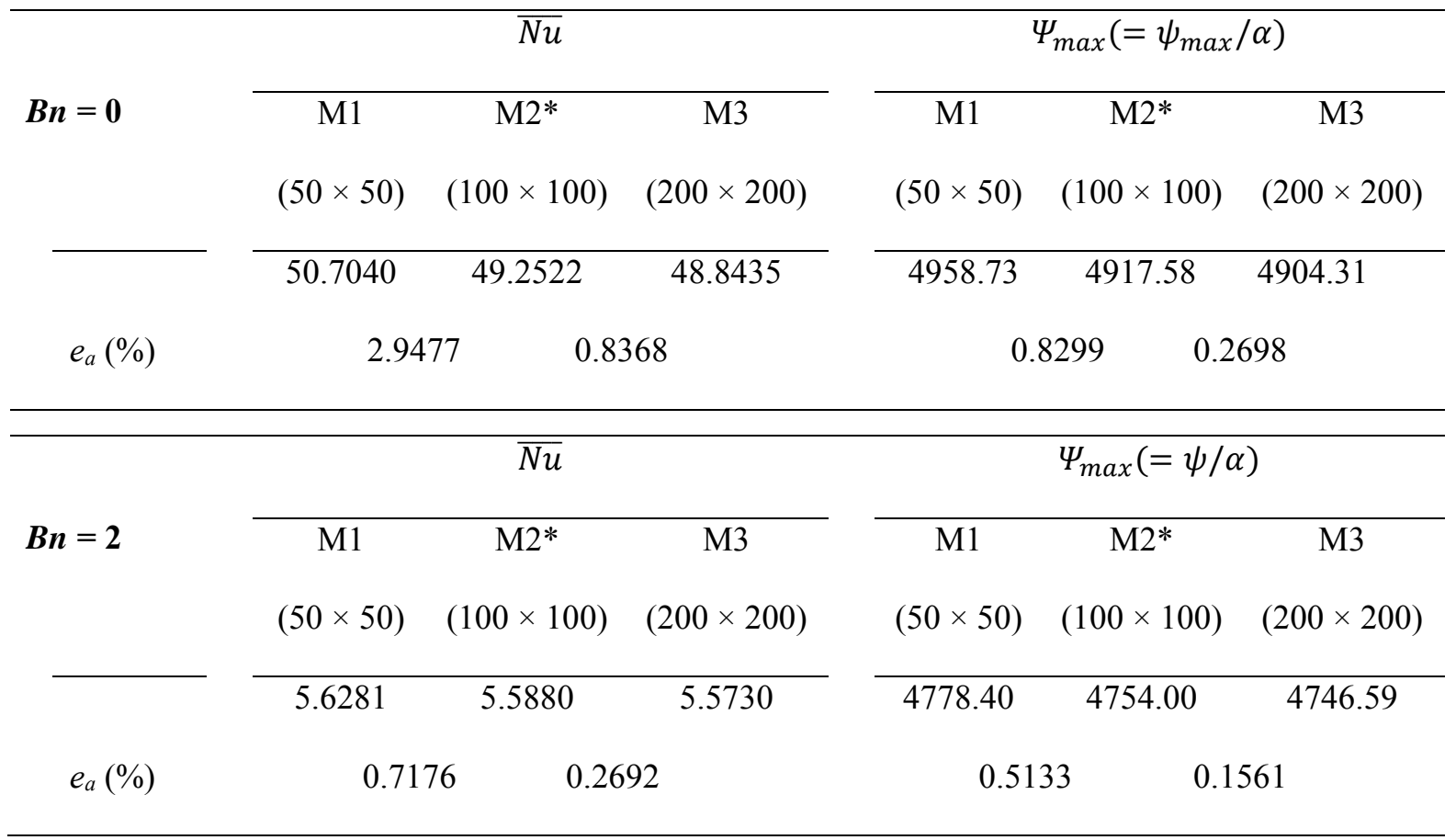

* The mesh that is used for the numerical simulations. 
Table 3. Scaling estimates for characteristic velocity scales.

\begin{tabular}{|c|c|c|c|}
\hline Velocity Scale & Forced Convection & Natural Convection & Mixed Convection \\
\hline$V \sim$ & $\Omega R^{*}$ & $\Omega R^{*}$ & $\Omega R^{*}$ \\
\hline$U \sim$ & $\Omega R^{*}$ & $\sqrt{g \beta \Delta T R^{* *}}$ & $a(\Omega R)+b(\sqrt{g \beta \Delta T R})^{* * * *}$ \\
\hline${ }^{*}[20],{ }^{* *}[25],{ }^{* * *} a=e^{-\theta R i}$ and $b=1-e^{-\theta R i}$ \\
\hline
\end{tabular}


Table 4. Summary of scaling analysis.

The balance between inertial and viscous forces $\rho \frac{V^{2}}{R} \sim \frac{\tau}{\delta}$

\begin{tabular}{|c|c|c|}
\hline \multirow{3}{*}{\multicolumn{2}{|c|}{ Velocity scales }} & \multirow{3}{*}{$\begin{array}{l}V \sim \Omega R \\
U \sim a(\Omega R)+b(\sqrt{g \beta \Delta T R})\end{array}$} \\
\hline & & \\
\hline & & \\
\hline \multicolumn{2}{|l|}{ Shear stress } & $\tau \sim \tau_{y}+\mu(U / \delta)$ \\
\hline \multicolumn{2}{|c|}{ Hydrodynamic boundary layer thickness } & $\frac{\delta}{\sim} \frac{B n}{1}+\frac{1}{R n^{2}+4\left[a R e+h\left(\frac{R a}{{ }^{2}}\right)^{1 / 2}\right]}$ \\
\hline \multirow{2}{*}{\multicolumn{2}{|c|}{ Nusselt number }} & \multirow{2}{*}{$\overline{N u} \sim \frac{2 R e}{B n+\sqrt{B n^{2}+4\left[a R e+b\left(\frac{R a}{P r}\right)^{1 / 2}\right]}} f_{1}$} \\
\hline & & \\
\hline \multirow{3}{*}{$B n \neq 0$} & $R i=0$ & $\overline{N u} \sim \frac{2 R e}{B n+\sqrt{B n^{2}+4 R e}} f_{1}$ \\
\hline & \multirow[b]{2}{*}{$R i \gg 1$} & \multirow{2}{*}{$\overline{N u} \sim \frac{2 R e}{B n+\sqrt{B n^{2}+4\left(\frac{R a}{P r}\right)^{1 / 2}}} f_{1}$} \\
\hline & & \\
\hline \multirow{2}{*}{$B n=0$} & $R i=0$ & $\overline{N u} \sim R e^{1 / 2} f_{1}$ \\
\hline & $R i \gg 1$ & $\overline{N u} \sim R i^{-1 / 2}(R a / P r)^{1 / 4} f_{1}=(R a / P r)^{1 / 4} f_{2}$ \\
\hline
\end{tabular}


Table 5. Values of $B n_{\max }$ at different values of $R i, R e$ and $P r$. The Bingham number at which $\overline{N u}=$ 1.0001 is referred to as $B n_{\max }$. For $B n>B n_{\max }$ one obtains $1 \leq \overline{N u}<1.0001$.

\begin{tabular}{rccc}
\hline \multicolumn{5}{c}{$\boldsymbol{R i}(=\mathbf{0}, \mathbf{0 . 1}, \mathbf{0 . 5}, \mathbf{1})$} \\
\hline $\boldsymbol{R e}$ & $\boldsymbol{P r}=\mathbf{1 0}$ & $\boldsymbol{P r}=\mathbf{1 0 0}$ & $\boldsymbol{P r}=\mathbf{5 0 0}$ \\
\hline 100 & 25 & 80 & 200 \\
500 & 125 & 400 & 1000 \\
1000 & 250 & 900 & 2500 \\
2000 & 500 & 1800 & 5000 \\
3000 & 750 & 3000 & 9000 \\
\hline
\end{tabular}


Table 6. Summary of the mean Nusselt number correlation for Newtonian fluids $(B n=0)$.

$$
\overline{N u}_{B n=0}=1+k_{0} R e^{m_{0}}
$$

$10 \leq \operatorname{Pr}<100$ and $0 \leq R i \leq 1$

$k_{0}=(0.015-0.003 \ln P r)+(0.113 \ln P r-0.164) \exp (-\operatorname{Ri}(2.539+1.214 \ln P r))$

$m_{0}=(0.746-0.022 \ln P r)+(0.367 \ln P r-0.83) R i^{(0.229 \ln P r-0.046)}$

$100 \leq \operatorname{Pr} \leq 500$ and $0 \leq R i \leq 0.5$

$k_{0}=(0.283 \ln P r-0.947) \exp (-R i(16.76-1.874 \ln P r))$

$\left.m_{0}=(0.742-0.02 \ln P r)+(1.65-0.172 \ln P r) R i^{(0.9+0.023 \ln P r}\right)$

$100 \leq \operatorname{Pr} \leq 500$ and $0.5<R i \leq 1$

$k_{0}=(0.065 \ln P r+0.012) \exp (-R i(4.187+0.161 \ln P r))$

$m_{0}=(-0.188+0.135 \ln P r)+(0.793-0.025 \ln P r) R i^{(0.107+0.137 \ln P r)}$ 
Table 7. Summary of the mean Nusselt number correlation for Bingham fluids.

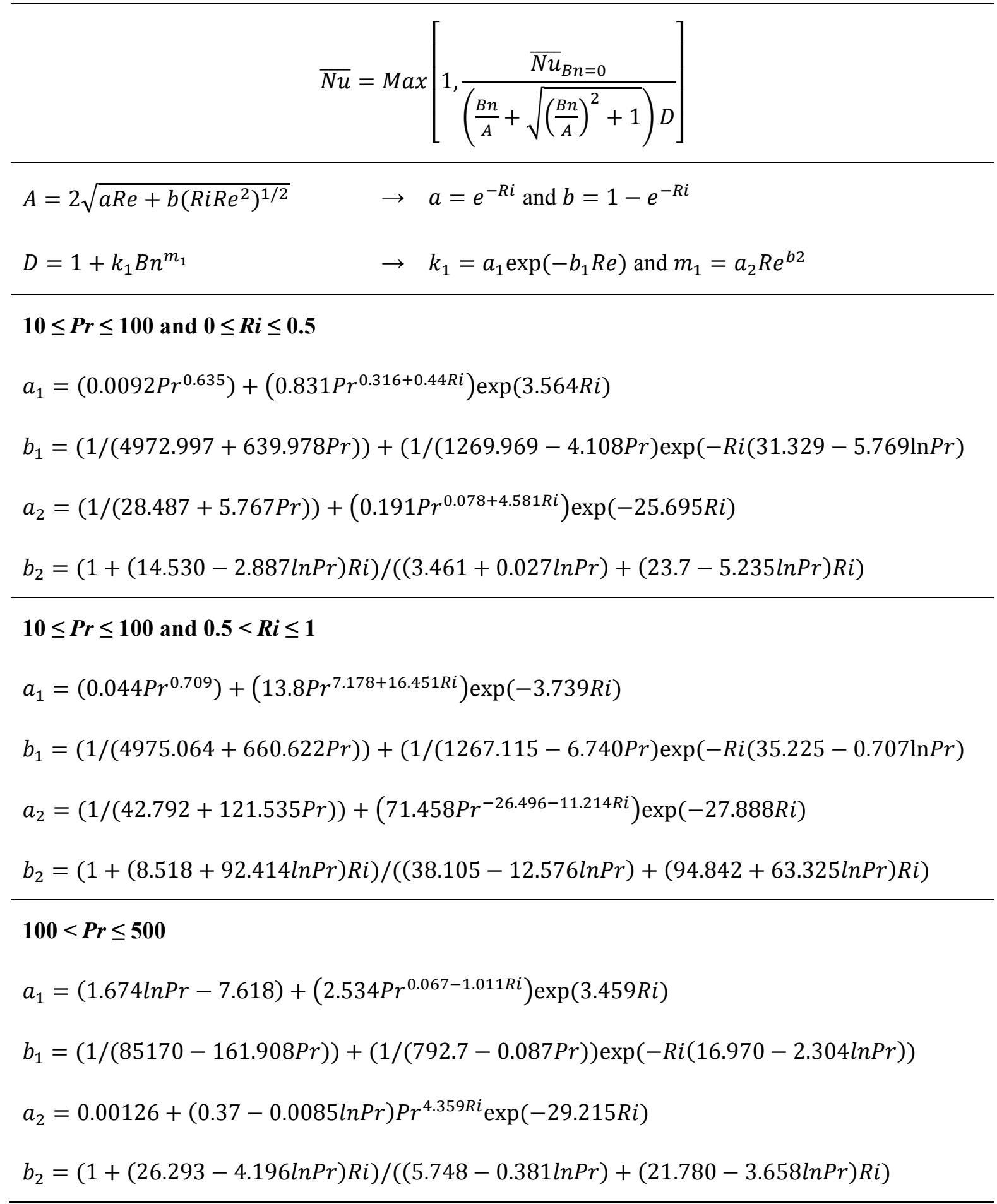




\section{FIGURE CAPTIONS}

Fig. 1. Schematic diagram of simulation domain and boundary conditions.

Fig. 2. The comparison of present simulation results for Newtonian fluid with benchmark results by Iwatsu [9] for $\operatorname{Pr}=1$.

Fig. 3. The variation of the mean Nusselt number $\overline{N u}$ with Bingham number $B n$ for $R i=0$ at $R e=500$ and $\operatorname{Pr}=500$.

Fig. 4. The interrelation between the mean Nusselt number $\overline{N u}$ and Bingham number $B n$ for different values of Reynolds number $R e$ for $R i=0$ (left column) and $R i=1$ (right column) at $P r=500$.

Fig. 5. The contours of non-dimensional temperature $\theta$ and stream function $\Psi=\psi / \alpha$ with AURs (shown in grey) for different values of $B n$ for $R i=0$ and $R i=1$ at $R e=2000$ and $P r=500$.

Fig. 6. The variation of mean Nusselt number $\overline{N u}$ with Reynolds number $R e$ in the case of Newtonian (i.e. $B n=0$ ) and Bingham (i.e. $B n=1$ and 5) fluids for $R i=0$ and 1 at $\operatorname{Pr}=500$.

Fig. 7. The variation of non-dimensional temperature $\theta$ and normalised swirl velocity component $V_{\theta} /$ $V_{\theta_{\text {max }}}$ along the vertical mid-plane (i.e. $\left.r / R=0.5\right)$ for different $R e$ values for Newtonian $($ i.e. $B n=0)$ and Bingham Fluid (i.e. $B n=1$ and 10) cases at $R i=0.1$ and $\operatorname{Pr}=500$.

Fig. 8. The variation of mean Nusselt number $\overline{N u}$ with Richardson number $R i$ for different values of Bingham number at $\operatorname{Re}=1000$ and $\operatorname{Pr}=500$.

Fig. 9. The variation of non-dimensional temperature $\theta$ and normalised swirl velocity component $V_{\theta} / V_{\theta \max }$ along the vertical mid-plane (i.e. $r / R=0.5$ ) for different $R i$ values for $B n=0,1,100$ at $R e=$ 1000 and $\operatorname{Pr}=500$.

Fig. 10. The variation of mean Nusselt number $\overline{N u}$ with Prandtl number $\operatorname{Pr}$ in the case of Newtonian (i.e. $B n=0$ ) and Bingham (i.e. $B n=1$ and 10) fluids for $R i=0$ and 1 at $R e=1000$ and $\operatorname{Pr}=500$.

Fig. 11. The variation of non-dimensional temperature $\theta$ and swirl velocity component $V_{\theta}$ along the vertical mid-plane (i.e. $r / R=0.5$ ) for different $\operatorname{Pr}$ values for Newtonian (i.e. $B n=0$ ) and Bingham Fluid (i.e. $B n=1$ and 10$)$ cases at $R i=1$ at $R e=1000$. 
Fig. 12. The variation of $B n_{\max }$ with Reynolds number $R e$ for different values of $\operatorname{Pr}$ and $R i$ along with the prediction of Eq. (33) (---).

Fig. 13. Comparison between $\overline{N u}$ obtained from the simulations with the predictions of Eq. (34) for the Newtonian fluid (i.e. $B n=0$ ) case.

Fig. 14. Comparison between $\overline{N u}$ obtained from the simulations with the predictions of Eq. (35) for the Bingham fluid case for different $R i, R e$ and $P r$.

Fig. 15. The interrelation between the torque coefficient $C_{T}$ and (a) Bingham number $B n$, (b) Reynolds number Re, (c) Richardson number $R i$ and (d) Prandtl number $P r$. 


\section{TABLE CAPTIONS}

Table 1. The normalised minimum grid spacing $\Delta_{\text {min,cell }} / R$ and grid expansion ratio $r_{e}$

Table 2. The numerical uncertainty for the mean Nusselt number $\overline{N u}$ and maximum non-dimensional stream function $\Psi_{\max }\left(=\psi_{\max } / \alpha\right)$ in case of Newtonian and Bingham fluids for $R e=3000$ and $R i=0$ at $\operatorname{Pr}=500$.

Table 3. Scaling estimates for characteristic velocity scales

Table 4. Summary of scaling analysis

Table 5. Values of $B n_{\max }$ at different values of $R i, \operatorname{Re}$ and $P r$. The Bingham number at which $\overline{N u}=$ 1.0001 is referred to as $B n_{\max }$. For $B n>B n_{\max }$ one obtains $1 \leq \overline{N u}<1.0001$.

Table 6. Summary of the mean Nusselt number correlation for Newtonian fluids $(B n=0)$.

Table 7. Summary of the mean Nusselt number correlation for Bingham fluids. 

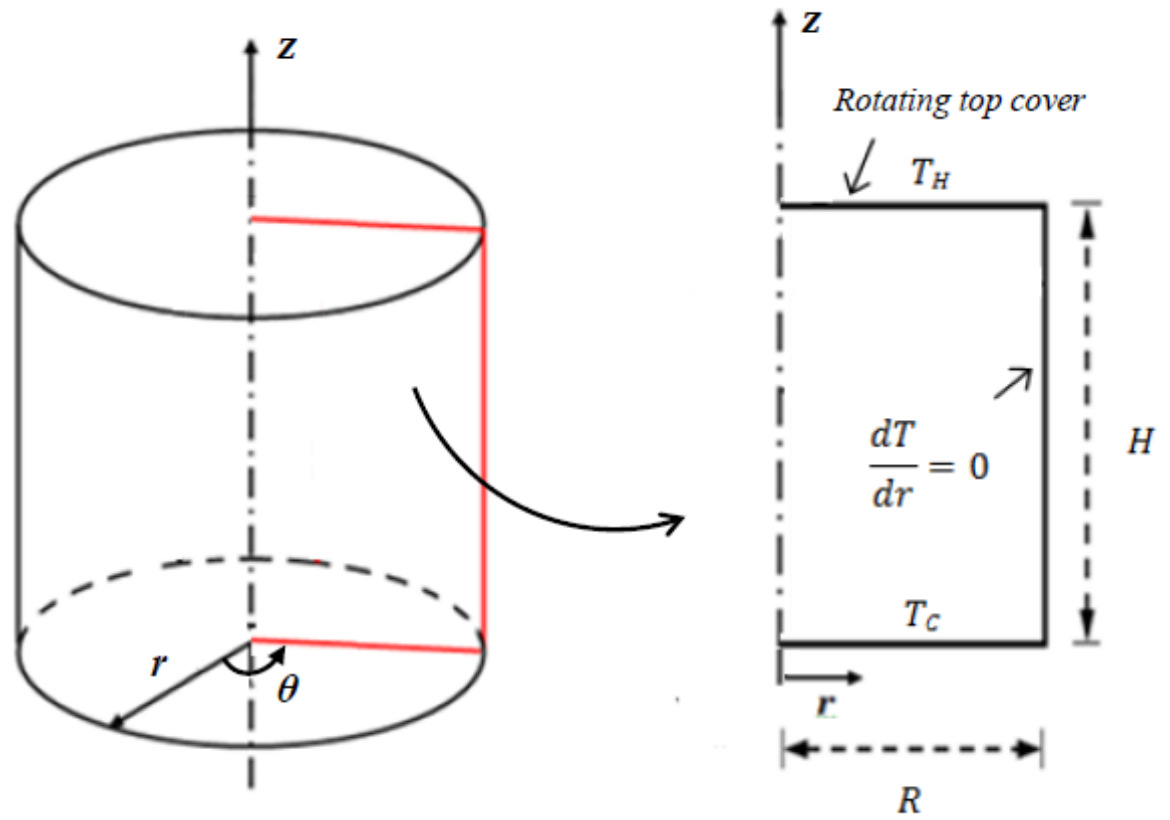

Fig.1. Schematic diagram of simulation domain and boundary conditions. 

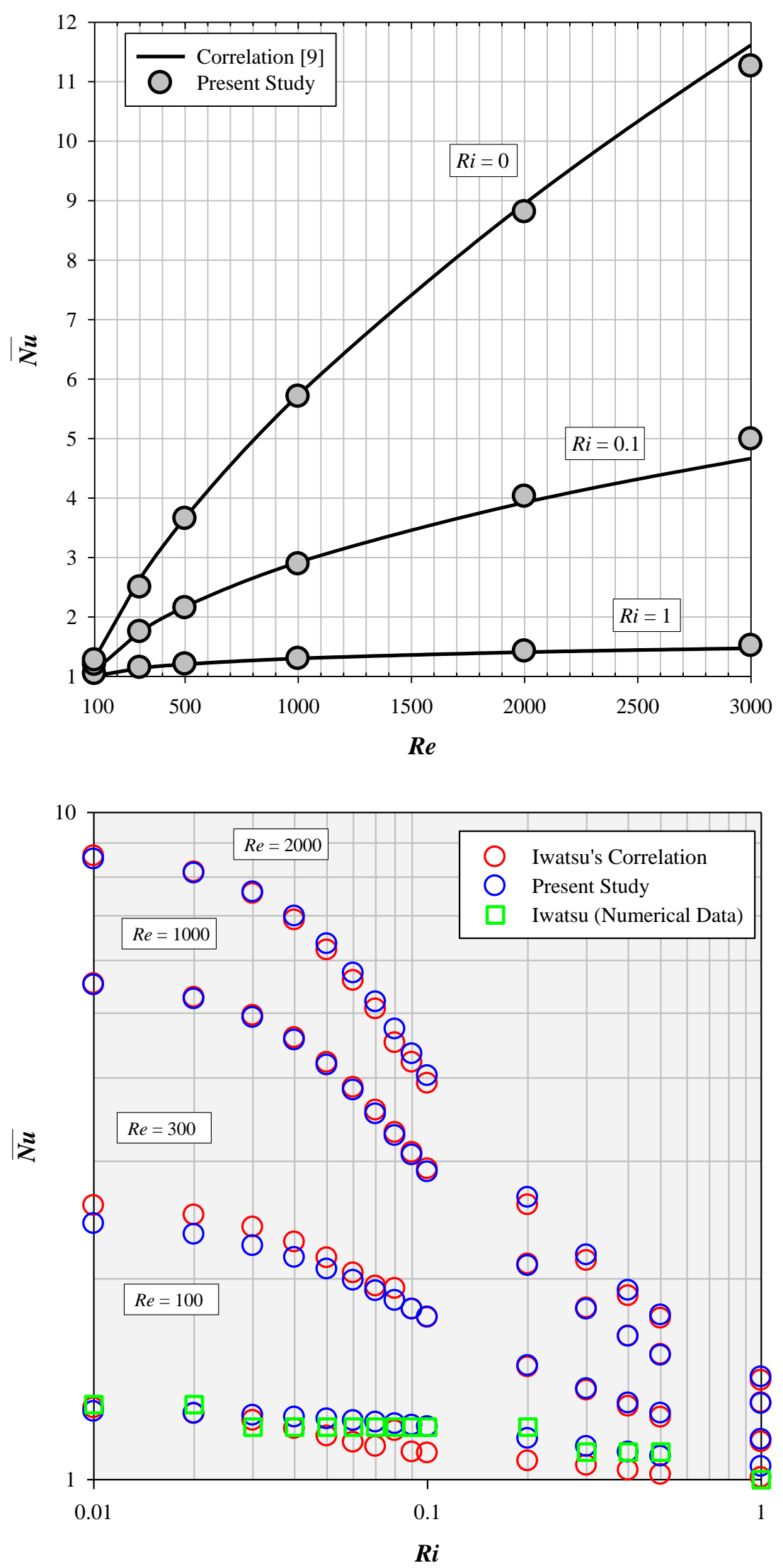

Fig. 2. The comparison of present simulation results for Newtonian fluid with benchmark results by Iwatsu [9] for $\operatorname{Pr}=1$. 

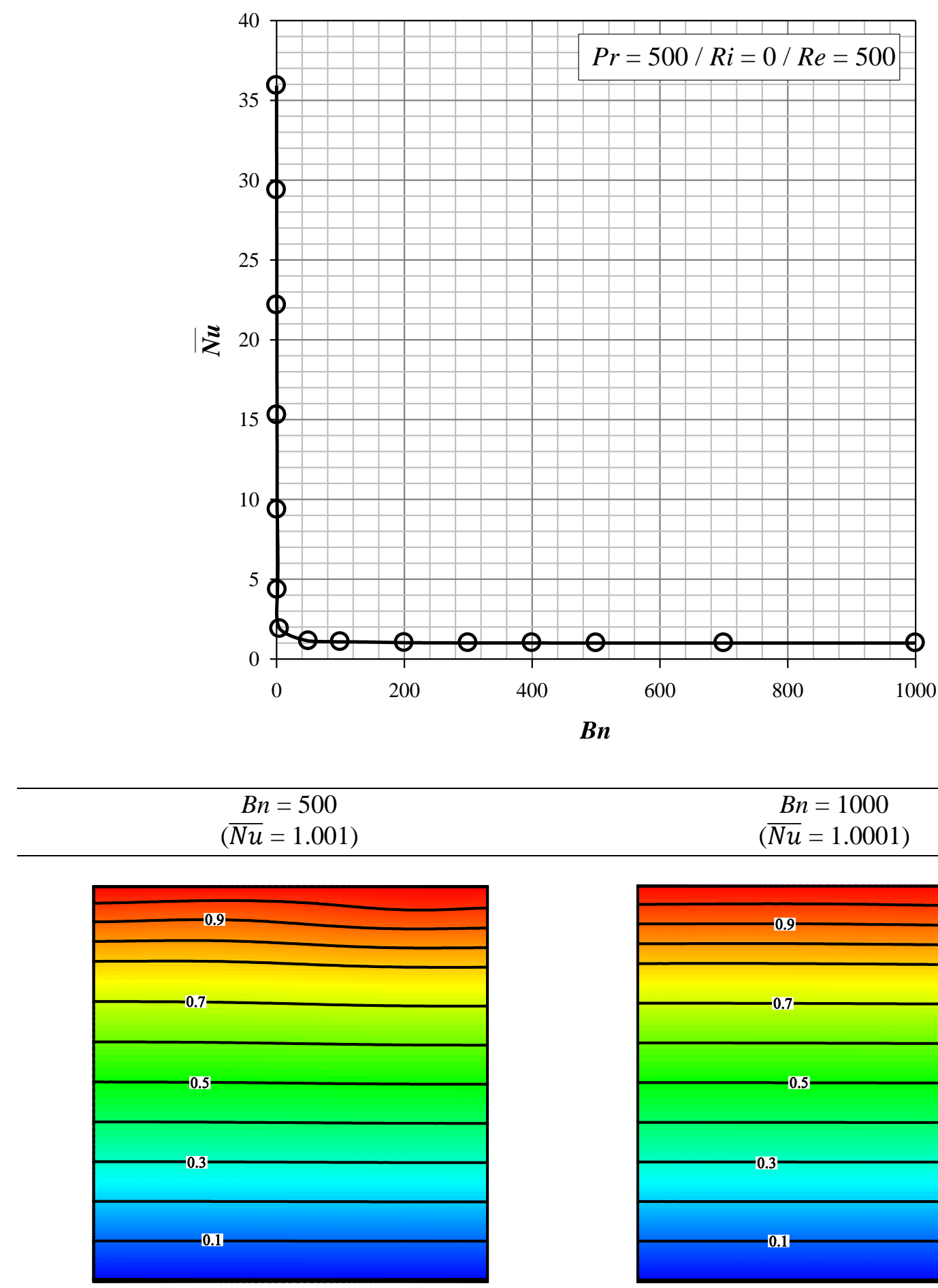

$$
\begin{gathered}
B n=1000 \\
(\overline{N u}=1.0001)
\end{gathered}
$$

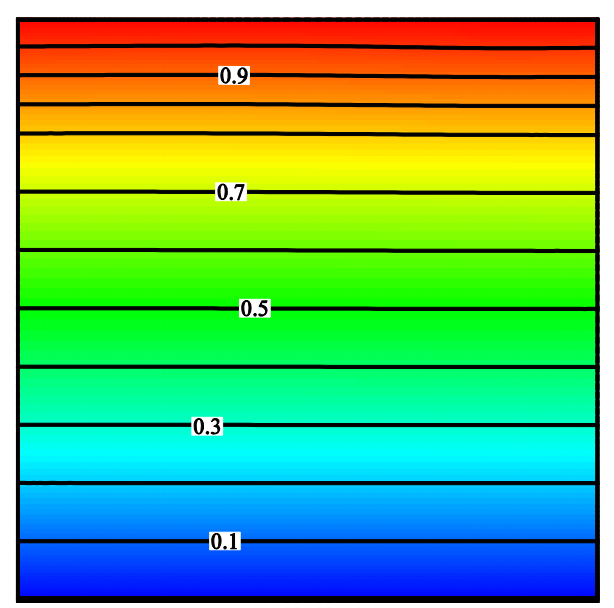

Fig. 3. The variation of the mean Nusselt number $\overline{N u}$ with Bingham number $B n$ for $R i=0$ at $R e=500$ and $\operatorname{Pr}=500$. 

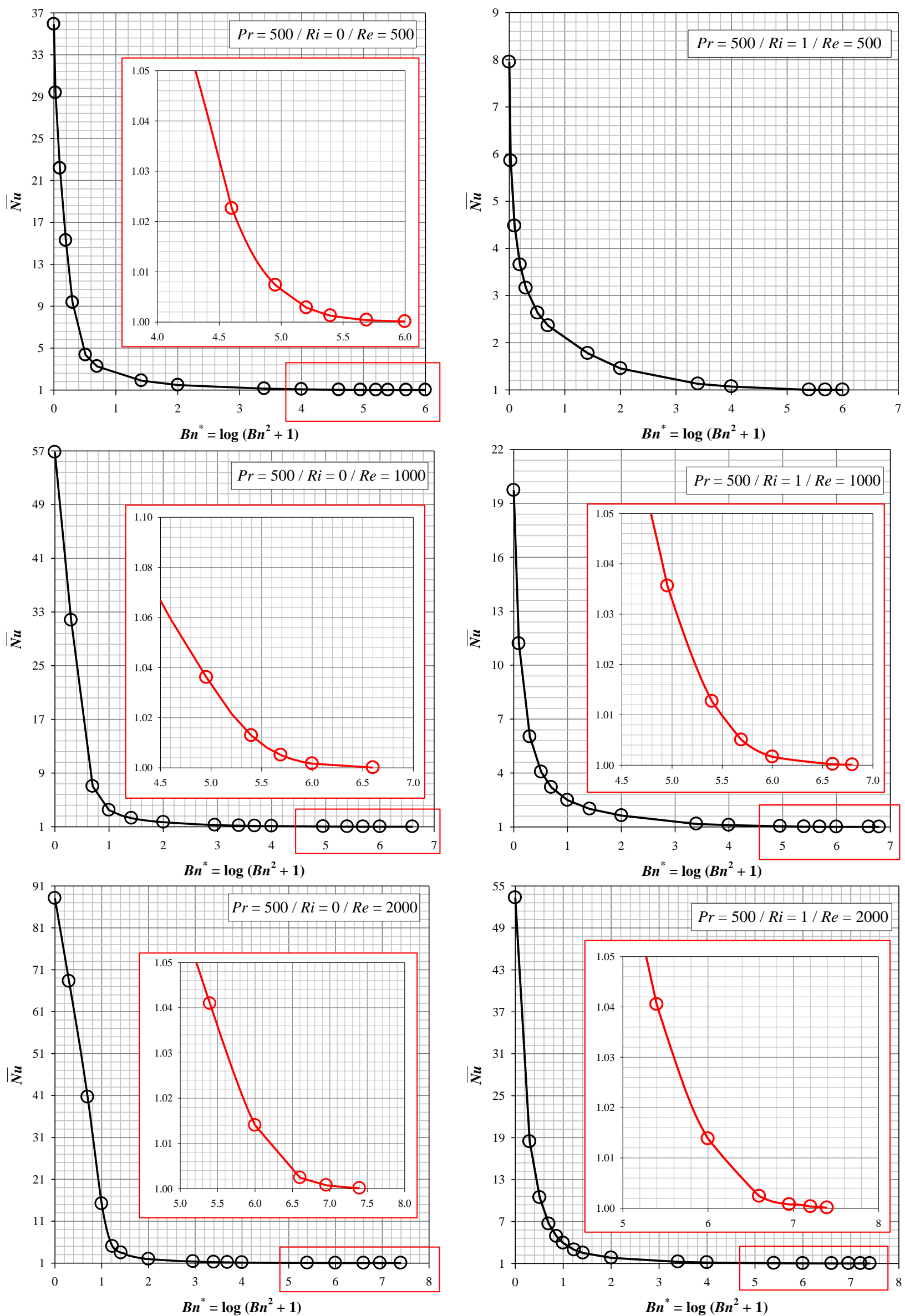

Fig. 4. The interrelation between the mean Nusselt number $\overline{N u}$ and Bingham number $B n$ for different values of Reynolds number $R e$ for $R i=0$ (left column) and $R i=1$ (right column) at $\operatorname{Pr}=500$. 


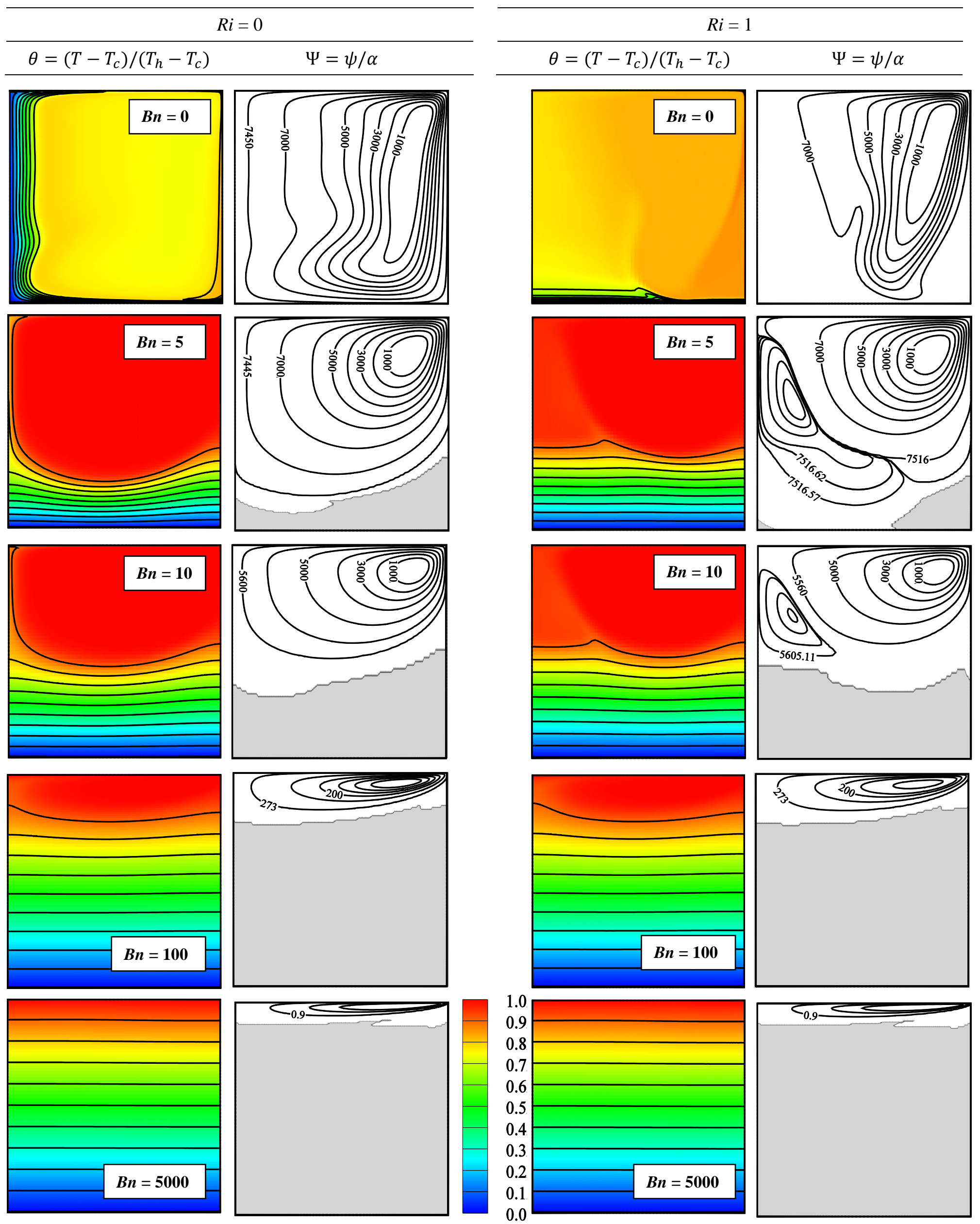

Fig. 5. The contours of non-dimensional temperature $\theta$ and stream function $\Psi=\psi / \alpha$ with AURs (shown in grey) for different values of $B n$ for $R i=0$ and $R i=1$ at $R e=2000$ and $P r=500$. 

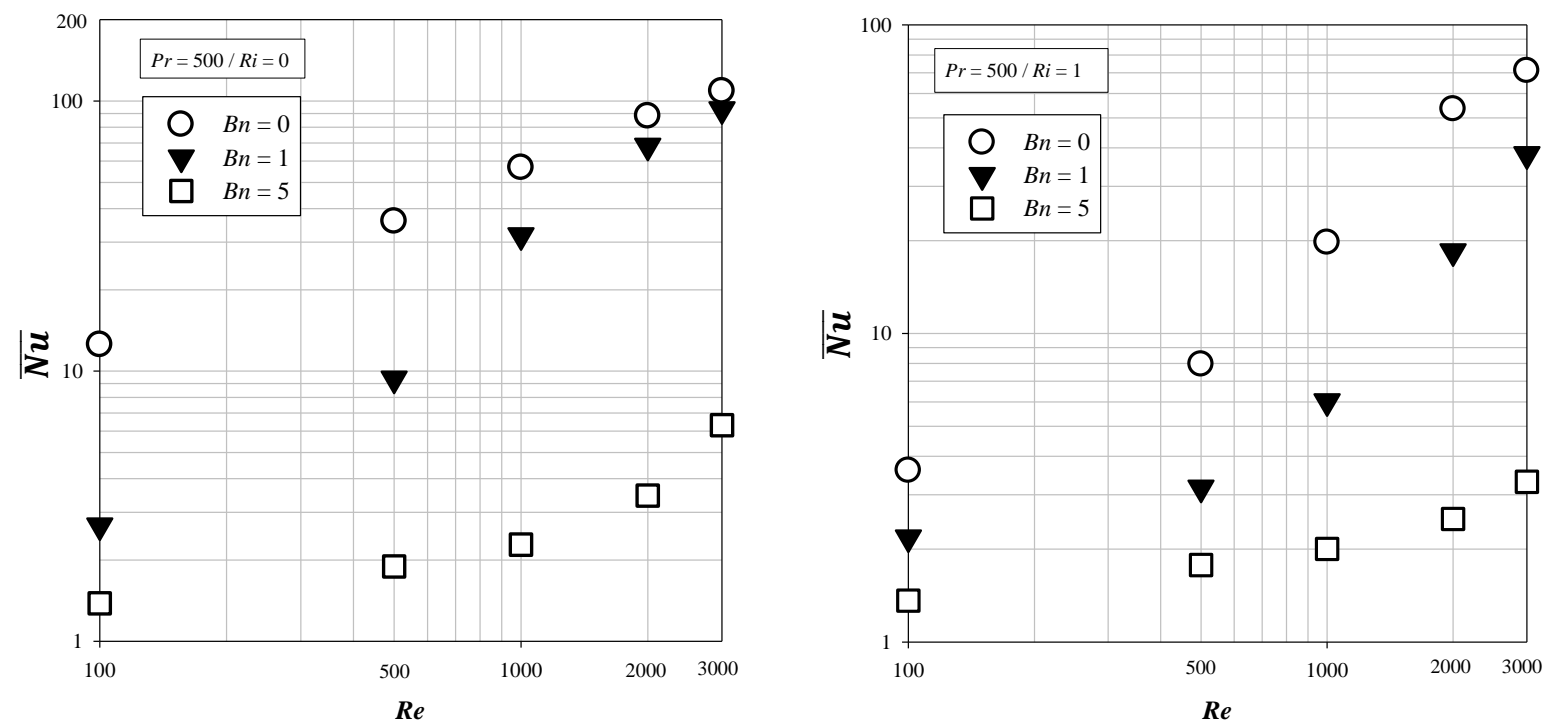

Fig. 6. The variation of mean Nusselt number $\overline{N u}$ with Reynolds number $R e$ in the case of Newtonian (i.e. $B n=0$ ) and Bingham (i.e. $B n=1$ and 5) fluids for $R i=0$ and 1 at $P r=500$. 

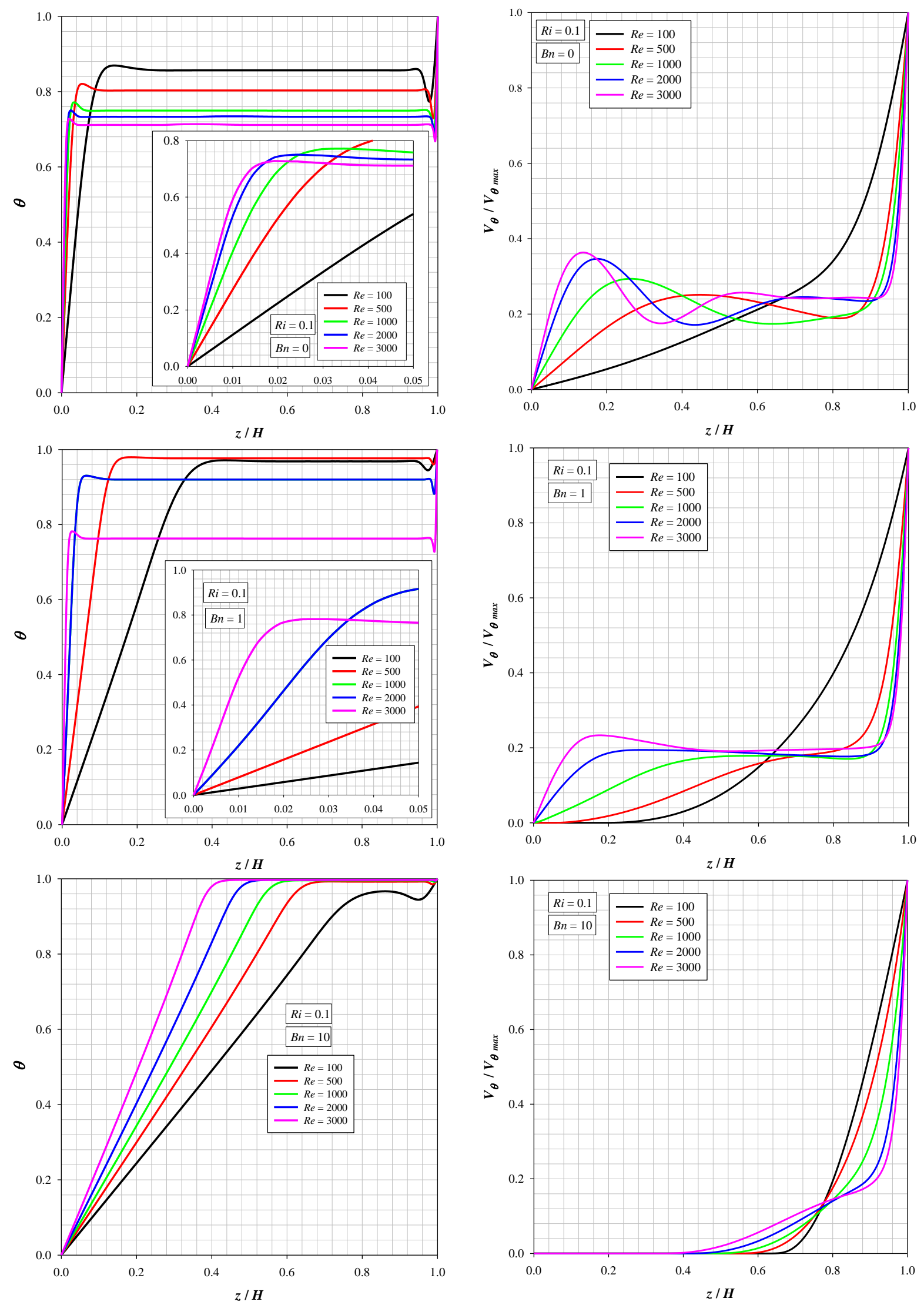

Fig. 7. The variation of non-dimensional temperature $\theta$ and normalised swirl velocity component $V_{\theta} / V_{\theta_{\max }}$ along the vertical mid-plane (i.e. $r / R=0.5$ ) for different $R e$ values for Newtonian (i.e. $B n=0$ ) and Bingham Fluid (i.e. $B n=1$ and 10) cases at $R i=0.1$ and $\operatorname{Pr}=500$. 


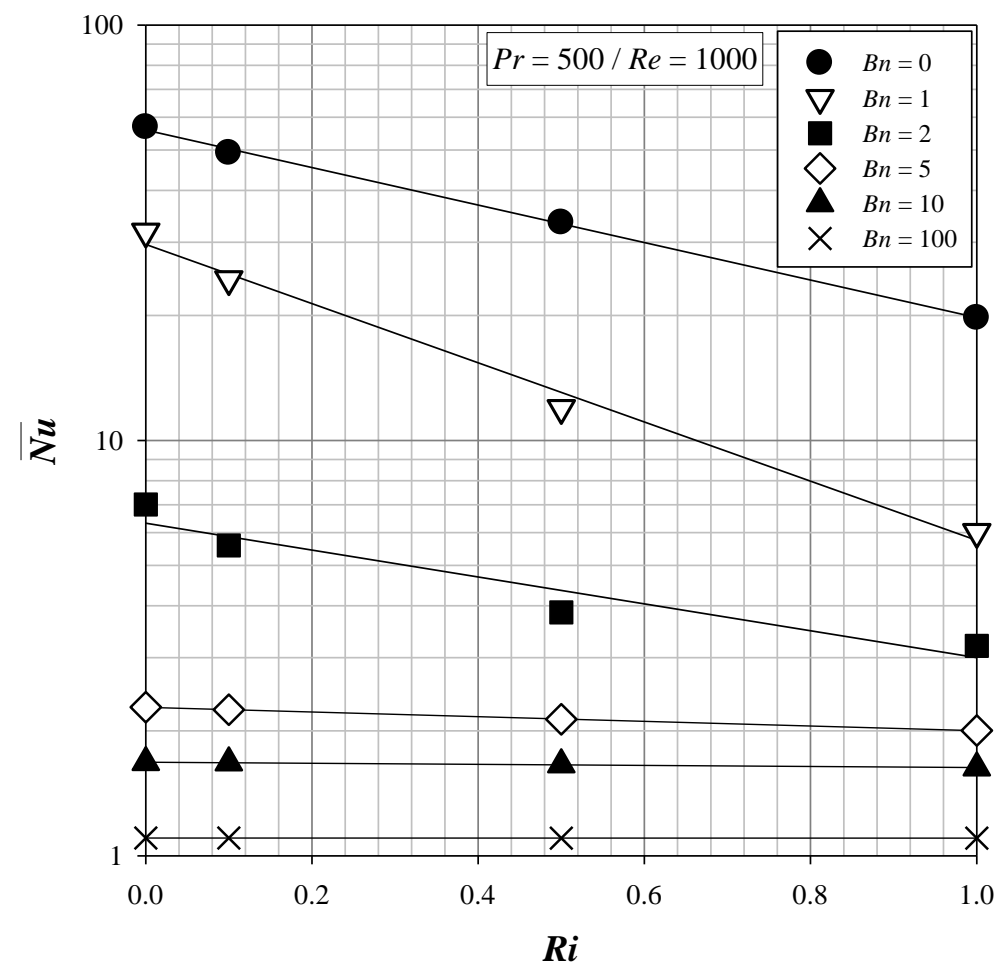

Fig. 8. The variation of mean Nusselt number $\overline{N u}$ with Richardson number $R i$ for different values of Bingham number at $\operatorname{Re}=1000$ and $\operatorname{Pr}=500$. 

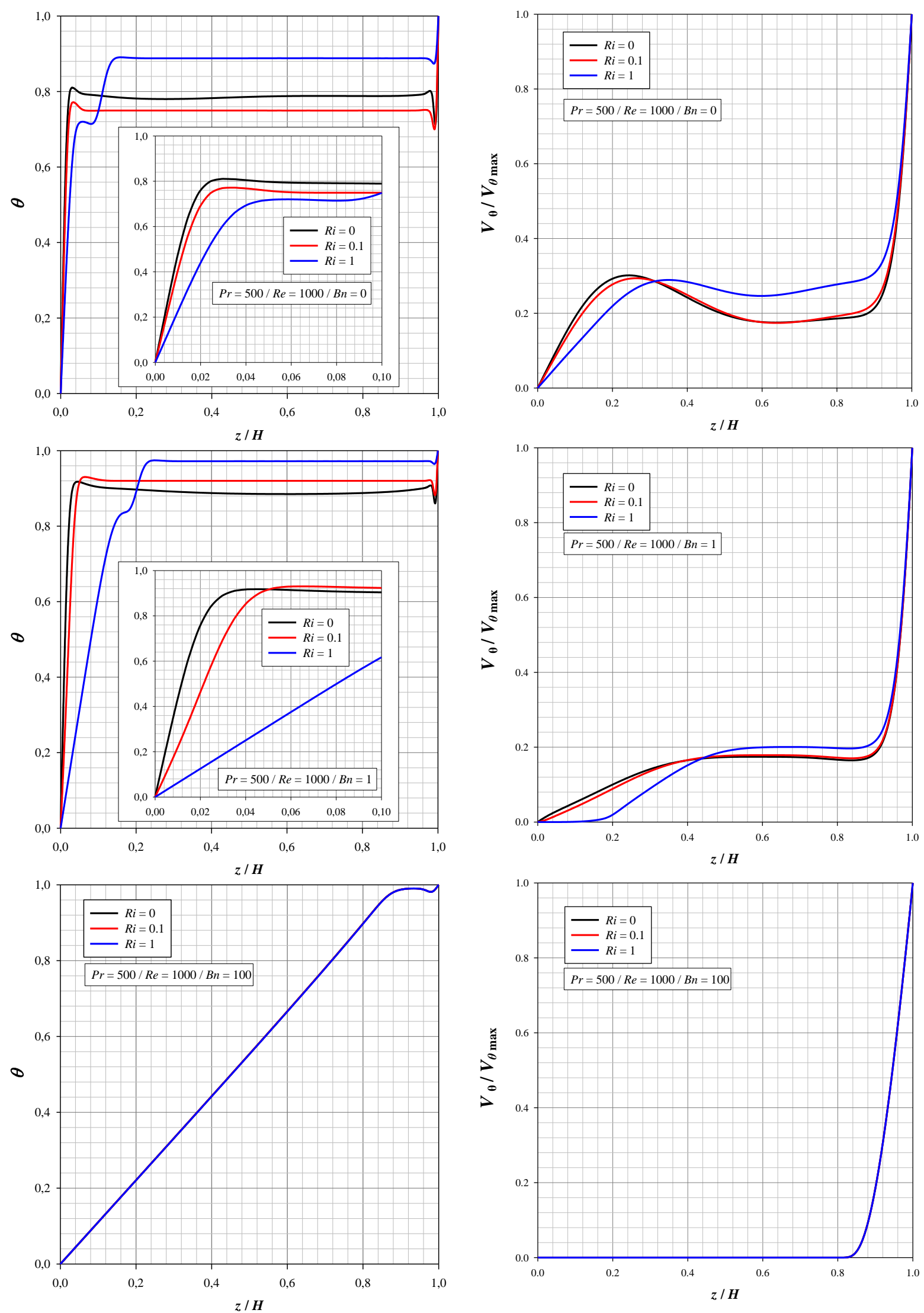

Fig. 9. The variation of non-dimensional temperature $\theta$ and normalized swirl velocity component $V_{\theta} / V_{\theta \max }$ along the vertical mid-plane (i.e. $r / R=0.5$ ) for different $R i$ values for $B n=0,1,100$ at $\operatorname{Re}=1000$ and $\operatorname{Pr}=500$. 

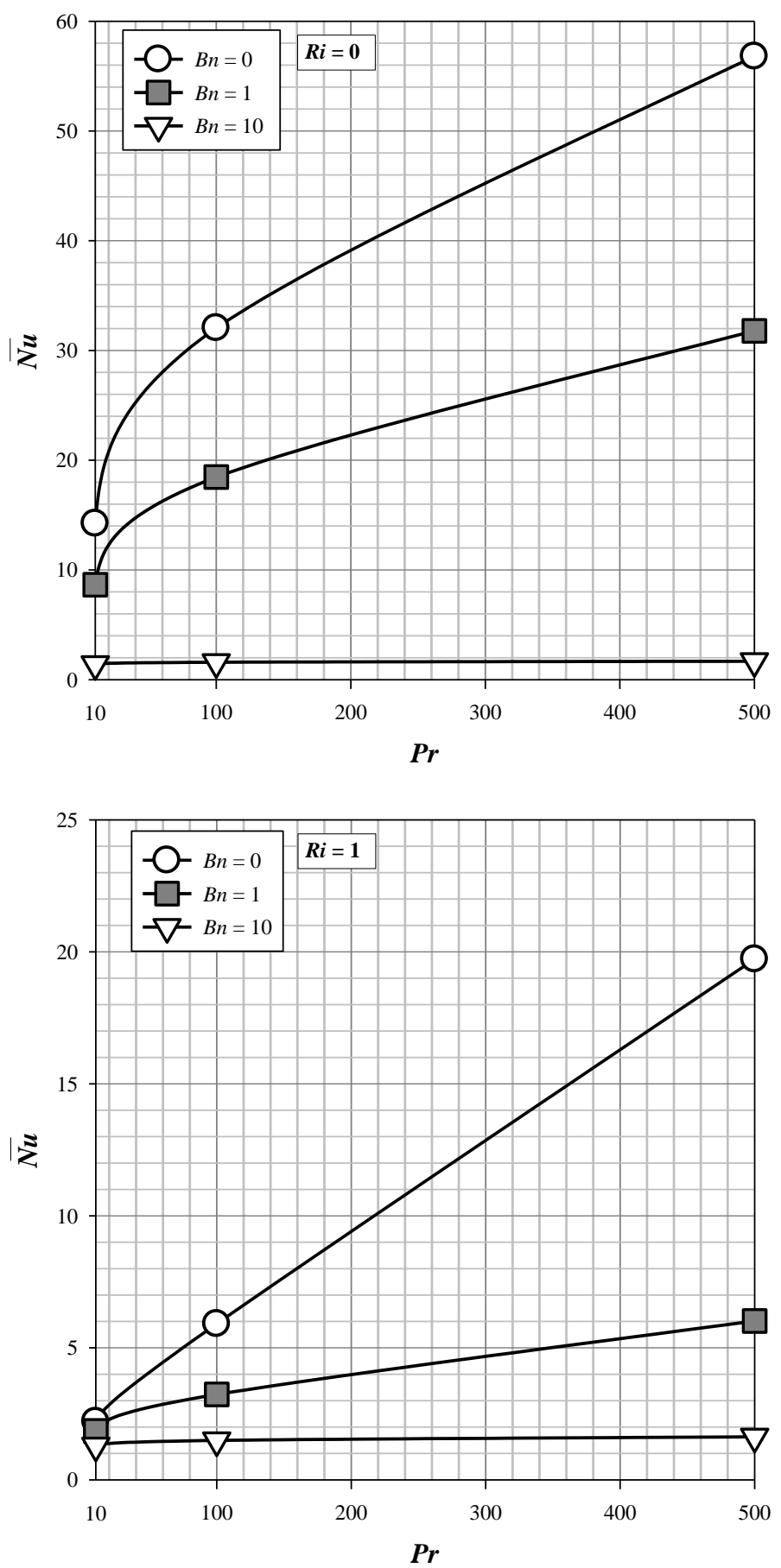

Fig. 10. The variation of mean Nusselt number $\overline{N u}$ with Prandtl number $\operatorname{Pr}$ in the case of Newtonian (i.e. $B n=0$ ) and Bingham (i.e. $B n=1$ and 10) fluids for $R i=0$ and 1 at $R e=1000$ and $P r=500$. 

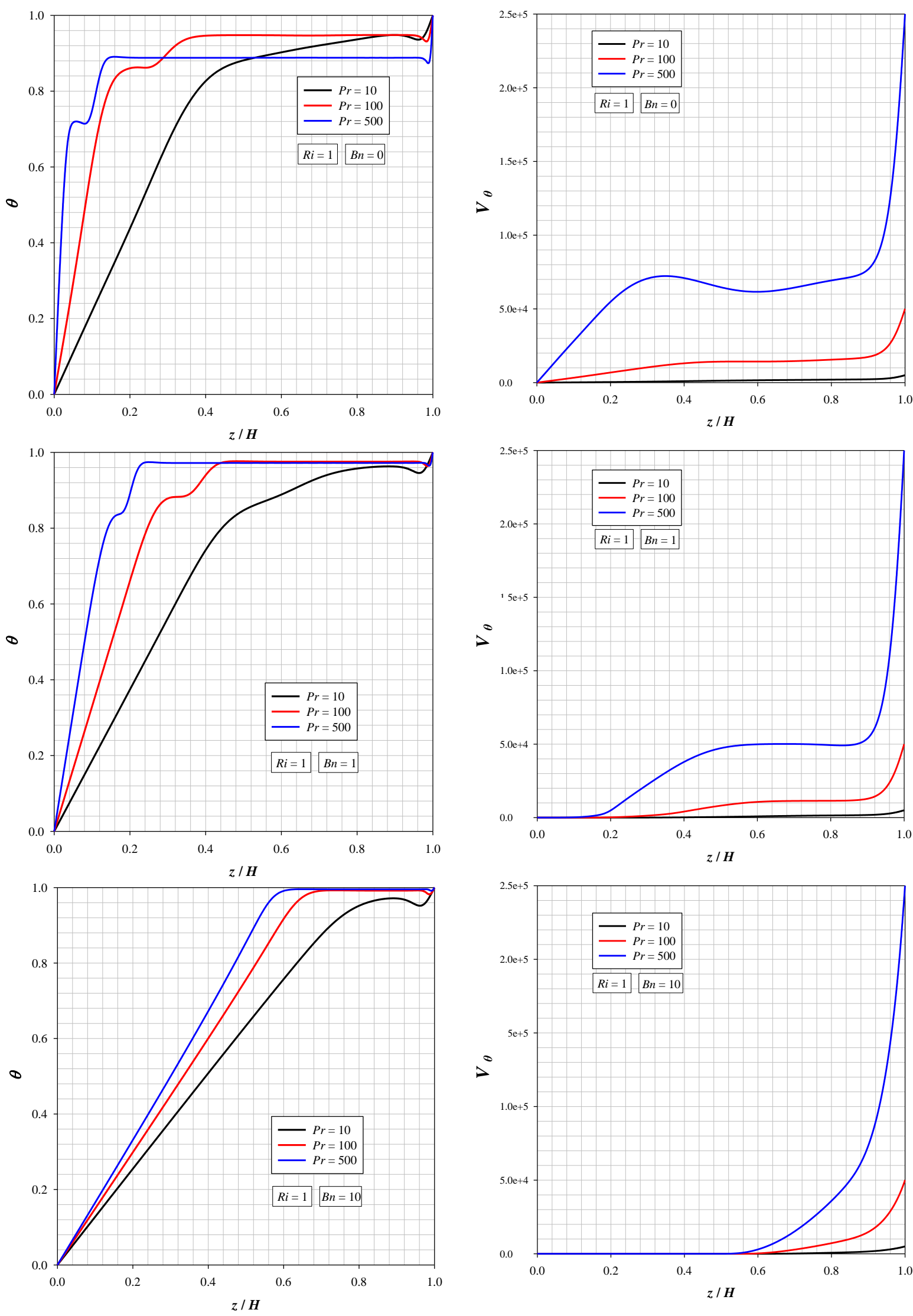

Fig. 11. The variation of non-dimensional temperature $\theta$ and swirl velocity component $V_{\theta}$ along the vertical mid-plane (i.e. $r / R=0.5$ ) for different $P r$ values for Newtonian (i.e. $B n=0$ ) and Bingham Fluid (i.e. $B n=1$ and 10 ) cases at $R i=1$ at $R e=1000$. 


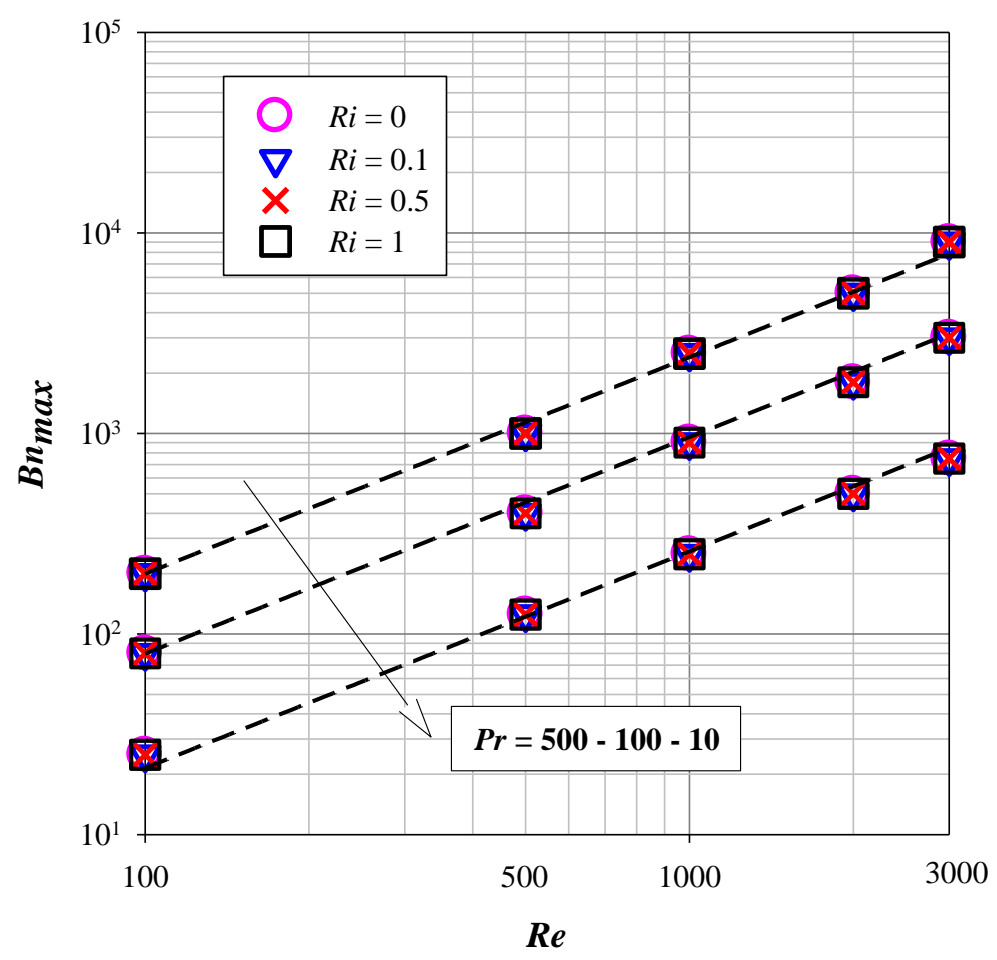

Fig. 12. The variation of $B n_{\max }$ with Reynolds number $R e$ for different values of $\operatorname{Pr}$ and $R i$ along with the prediction of Eq. (33) (---). 

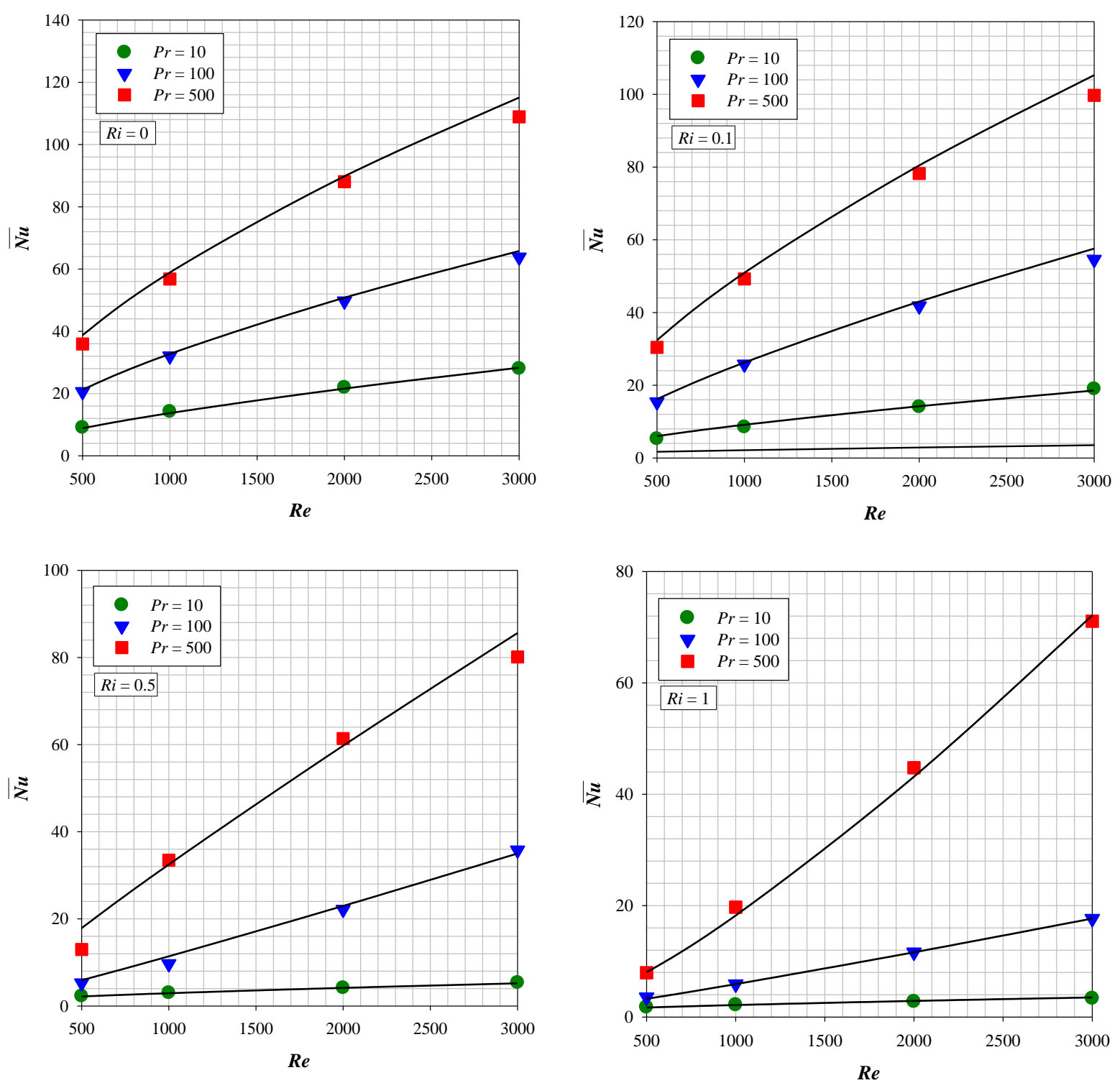

Fig. 13. Comparison between $\overline{N u}$ obtained from the simulations with the predictions of Eq. (34) for Newtonian fluid (i.e. $B n=0$ ) case. 

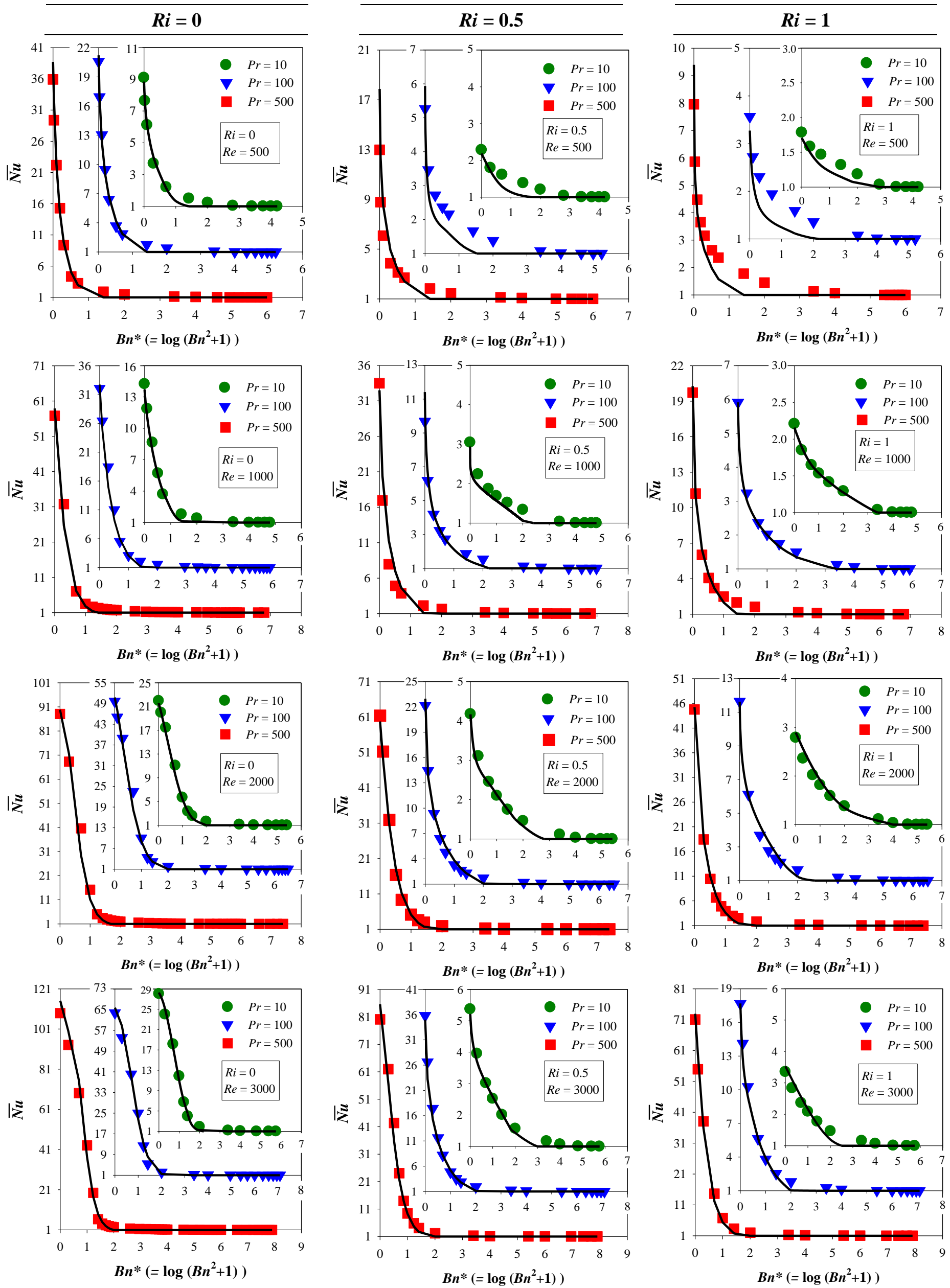

Fig. 14. Comparison between $\overline{N u}$ obtained from the simulations with the predictions of Eq. (35) for Bingham fluid case for different $R i, R e$ and $P r$. 

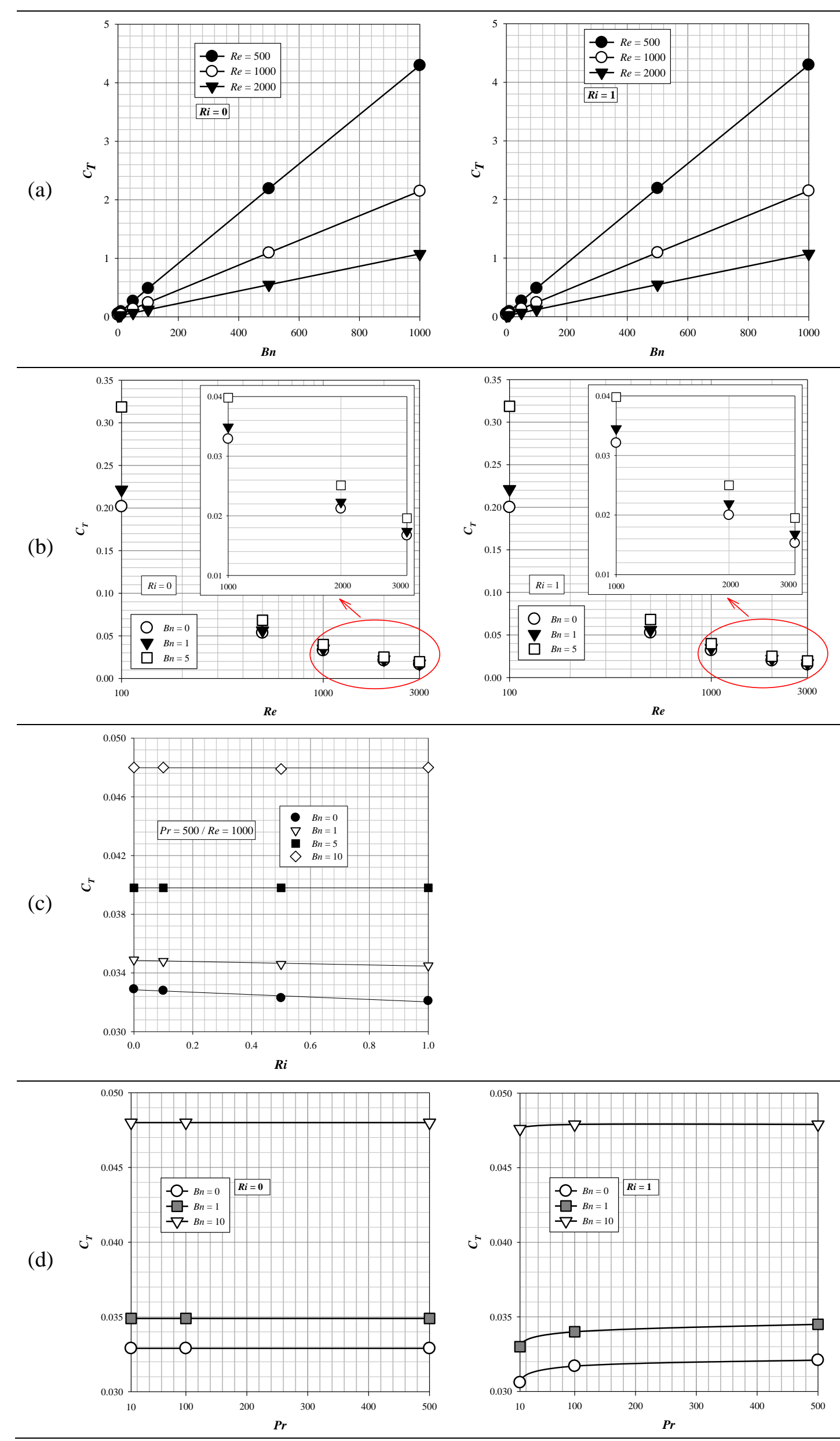

Fig. 15. The interrelation between the torque coefficient $C_{T}$ and (a) Bingham number $B n$, (b) Reynolds number Re, (c) Richardson number Ri, (d) Prandtl number Pr. 